\title{
Duration of courtship effort as a costly signal
}

\author{
Robert M. Seymour ${ }^{1,2,3}$ and Peter D. Sozou ${ }^{4}$ \\ ${ }^{1}$ Department of Mathematics \\ ${ }^{2}$ Centre for Mathematics and Physics in the Life Sciences and Experimental Biology \\ (CoMPLEX) \\ ${ }^{3}$ Centre for Economic Learning and Social Evolution (ELSE) \\ UCL \\ Gower Street \\ London WC1E 6BT \\ United Kingdom \\ Tel: $\quad+44(0) 2076792858$ \\ Fax: $\quad+44(0) 2073835519$ \\ Email: $\underline{\text { rms@math.ucl.ac.uk }}$ \\ ${ }^{4}$ Centre for Philosophy of Natural and Social Science \\ London School of Economics \\ Houghton Street \\ London WC2A 2AE \\ United Kingdom \\ Email:p.sozou@1se.ac.uk
}




\section{Abstract}

We consider a male and a female in a courtship encounter over continuous time. Both parties pay participation costs per unit time. The game ends when either one or other of the parties quits or the female accepts the male as a mate. We assume that there is a binary variable which determines whether the male is a "good" or "bad" type from the female's point of view, according to either his condition or his willingness to care for the young after mating. This variable is not directly observable by the female, but has fitness consequences for her: she gets a positive fitness payoff from mating with a "good" male but a negative fitness payoff from mating with a "bad" male. We assume also that a "good" male has a higher ratio of fitness benefit from mating to fitness cost per unit time of courtship than a "bad" male. We show that, under suitable assumptions, there are evolutionarily stable equilibrium behaviours in which timeextended courtship takes place. A "good" male is willing to court for longer than a "bad" male; in this way the duration of a male's courtship signals his type, and acts as a costly handicap. By not being willing to mate immediately the female achieves a degree of screening because the posterior probability that the male is "good", conditional on his not having quit the game, increases with the duration of courtship.

\section{Introduction}

In most species the reproductive success of males is largely governed by the ability to secure matings with receptive females (see for example Odendaal et al., 1985; Andersson and Iwasa, 1996), and increases with the number of females they mate with. Females, in contrast, invest greater resources into nurturing offspring, which limits the number of offspring they can produce; their reproductive success may depend to a significant extent on the quality of the males they mate with. This 
consideration may be sufficient for females to be choosy about which males they mate with even when delaying mating involves a cost (Moore and Moore 2001, Yamanoi et al., 2006).

Active female choice gives rise to selection pressure on male physiological and behavioural traits that influence a male's chance of being chosen by females, leading to the evolution of male signalling strategies (Dawkins, 1993; Johnstone, 1997). As in many signalling problems there is scope for deceit (Krebs and Dawkins 1984); this has the potential to reduce the credibility of signals. The seminal work of Spence (1973) showed that if a signal has the property that the benefit from signalling, relative to the cost, depends on the signaller's type, then a stable game-theoretic equilibrium may be possible in which signals tend to be informative to the receiver. In biology this notion leads to the 'handicap principle' (Zahavi and Zahavi, 1997), giving rise to evolutionarily stable outcomes in which superior males, from the point of view of a potential female mate, produce signals that are difficult or costly for inferior males to replicate (Grafen 1990a).

Courtship signalling by males has a variety of forms including adornments, bright colouration and extravagant displays (Andersson, 1994). Males may offer material gifts (e.g. Alcock, 1998; Vahed, 1998; Miller, 2000, Kondoh, 2001, Pizzari, 2003; Tryjanowski and Hromada, 2005). The female may obtain direct fitness benefits from such gifts in the form of enhanced nutrition for herself or direct investment in the provisioning of offspring (Gwynne, 1984). Edible nuptial gifts however, are not always of significant nutritional value to the female (Wedell, 1993). Gifts may also consist of inedible tokens (Vahed, 1998; Stalhandske, 2001, 2002). Even when 
nutritionally valuable gifts are offered in the majority of cases, offering a worthless gift can still be a successful strategy (LeBas and Hockam, 2005). In some circumstances, sending a signal with small functional benefits for the receiver may be advantageous to the signaller by giving the receiver added incentive to pay attention to the signal (Roberts, 1998). In contrast, Sozou and Seymour (2005), modelling courtship as a sequential game, argue that there is an important role for gifts that are costly to the male but worthless to the female. Because the gift is worthless to the female (and in the model she incurs a cost in accepting it), the male will not be exploited by a 'gold digger' who has no intention of mating with him. This fits within a sexual selection framework in which the male pays a cost of signalling to the female, and the female also pays a cost of screening the male (Pomiankowski, 1987).

In many cases, however, courtship involves not a single, instantaneous gift or act, but rather ongoing expenditure over a period of time. Courtship feeding in birds may involve several episodes before and after copulation (Nisbet, 1973). In black-horned tree crickets the duration of courtship feeding varies between males and appears to influence the number of fertilisations achieved by a male (Brown and Kuns, 2000). The Blue Bird of Paradise displays to a female by hanging upside down and vocalising for a prolonged period of time (Frith and Beehler, 1998). In modern human societies courtship typically involves a series of dinners and other outings. As well as direct costs to the male, these activities cost time and may therefore involve a significant opportunity cost to both sexes, i.e. a loss of the use of the time for other purposes. 
Where long courtship involves a net cost to the female, this raises the question: why does she not avoid (or reduce) this cost by deciding immediately (or more quickly) whether or not to mate with the male? It may be that a female cannot perfectly assess the male's quality because of random errors in the signal (Luttbeg 1996); with more observation time or repeat observations these random errors are reduced. There is, however, another possibility: that the duration of a male's courtship effort may in itself constitute a useful signal to the female. It is this possibility which the present study addresses.

We consider courtship as a game extended over time. With respect to post-mating payoffs the game is similar to that considered by Sozou and Seymour (2005). In particular males are always willing to mate but differ in some trait - genetic quality, or ability or willingness to provide paternal care - necessitating choosy behaviour by females. However, whereas Sozou and Seymour (2005) consider a single gift at a specific point in time, in the present study the courtship process involves a gradual accumulation of costs to both the male and the female over time. This can be conceptualised as a cycle of the male giving a small gift to the female repeated over many rounds, with a net cost to both parties over each cycle (i.e. the cost of the female's time exceeds the value of the gifts to her). However, the model considered here does not require that the courtship process necessarily involves "gifts" as such. Instead, each cycle can simply involve some form of costly act by the male, for example a courtship display, directed specifically at the female involved in the encounter (not a broadcast advertisement to females in general); the female also incurs some cost for participating in the process (e.g. a time-cost while she is observing the male). 


\section{The model}

\subsection{Representing fitness consequences of a courtship encounter}

A female and a male meet and engage in a courtship encounter which may lead to mating resulting in the production of offspring. Payoffs represent the fitness gain or loss arising from the encounter. We explicitly model only a single encounter, but this should be understood as an episode within an underlying biological process in which males and females may experience several encounters in the course of a breeding period. A player gaining a payoff of exactly zero is neither better off nor worse off than if the encounter had not taken place. That is, any player may gain or lose from a courtship encounter relative to their prospective outside options. The expected payoffs from such options can therefore be set to zero when analyzing a single (representative) encounter. A positive payoff represents a fitness gain from the encounter and a negative payoff represents a fitness loss. Thus if the female gets a negative payoff from mating, this means that she would have done better not to mate on that encounter but instead to hold out for the prospect of mating with a better male in a future encounter.

\subsection{Male type}

We assume that there is a variable which characterizes the male but which is not known a priori to the female. This can be regarded as the male's type. In common with Sozou and Seymour (2005), for simplicity we will consider this to be a binary variable. The male's type is either "good" $(G)$ or "bad" $(B)$. The case of an arbitrary 
number of male types is considered in Appendix C, where it is shown that the result is essentially the same as for the binary case.

We assume that a female gets a positive payoff from mating with a good male, and a negative payoff from mating with a bad male. We assume that a male always gets a positive payoff from mating, but that the ratio of his gain from mating to his cost of courtship is higher for a good male: that is, courtship effort is, relatively speaking, more costly for a bad male. These assumptions are presented more formally in section 2.3.

The most straightforward interpretation of a male's type is that it is a measure of his condition: a good male is in good condition; a bad male is in poor condition. A female should prefer to mate with a male in good condition if male condition is positively correlated with genetic quality, or in species with paternal care, a female should prefer to mate with a male in good condition if such a male is a better provider of care. If a male in good condition additionally has a lower cost of producing a strong courtship signal relative to his gain from mating, then the signal can act as an honest indicator of his condition, and a female should prefer to mate with a male producing a strong courtship signal (Parker, 1982; Grafen, 1990a; Grafen 1990b). In the model presented here, the duration of a male's courtship effort constitutes the strength of his signal.

An alternative interpretation of male type, proposed by Sozou and Seymour (2005), is modelled on species with facultative post-mating paternal care, e.g. humans. It is assumed that the male makes a subjective assessment of the female's attractiveness, 
and finds her either attractive, or unattractive. The female does not know for sure her own attractiveness to the male. The female's attractiveness to the male is a noisy indicator of her quality. The male values the chance of mating with her more highly if she is attractive to him, and in this case he will stay after mating and help raise the offspring. If she is unattractive he will still mate with her if given the opportunity, but will then desert, resulting in a negative payoff to the female. In this interpretation, a male discriminates between females: from his perspective they are not all the same. A female should accordingly discriminate between males on the basis of their intentions towards her (Camerer, 1988).

These interpretations of the game are mathematically equivalent in this model. The key features of the payoff structure of the game are:

1. The female gets a positive payoff from mating with a good male and a negative payoff from mating with a bad male.

2. A good male values mating with the female, relative to the costs of courtship, more highly than a bad male.

We assume that the female has objective, internalised prior probabilities for male type, determined by natural selection, and possibly by a limited degree of juvenile learning. The prior probability that a (random) male will be good $(G)$ is $P_{f}$, and $1-P_{f}$ is the probability that he will be bad $(B)$. We assume that $0<P_{f}<1$. 


\subsection{Payoffs from mating}

Following Sozou and Seymour (2005), we assume that if mating takes place, the fitness payoffs to both players will depend on the male's type. A male gets a higher payoff if he is $\operatorname{good}(G)$ rather than "bad" $(B)$.

Let $K=G$ (good) or $B$ (bad) be the type of the male. The male and female expected payoffs from mating are denoted by $M(K)$ and $F(K)$, respectively. We define the possible payoffs by:

$$
\begin{array}{ll}
M(G)=A_{m}, & M(B)=D_{m}, \\
F(G)=A_{f}, & F(B)=-C_{f},
\end{array}
$$

where we assume:

$$
\begin{aligned}
& A_{m}>D_{m}>0, \\
& A_{f}>0, C_{f}>0 .
\end{aligned}
$$

Thus, the female obtains a positive payoff from mating only if the male's type is good $(1 b, 2 b)$; if his type is bad, her payoff from mating with him is negative. However, any male will always mate with a female if given the opportunity because he always gets a positive payoff, though this payoff is higher if his type is good (1a, 2a) ${ }^{1}$.

\footnotetext{
${ }^{1}$ In this formulation we have assumed that the male gets a higher benefit from mating if he is a good type, but he pays the same cost of courtship per unit time whether he is a good or bad type. An
} 
If the female mates with a random male without any further information beyond the prior probability of his type being good, her expected payoff is

$$
E_{\text {prior }}^{F}=P_{f} A_{f}-\left(1-P_{f}\right) C_{f} .
$$

This may or may not be positive. If it is negative, the female cannot benefit from mating with the male, and she will refuse to do so (i.e. quit the encounter immediately), and receive zero payoff. If $E_{\text {prior }}^{F}$ is positive, she can receive this expected payoff by mating immediately. If $E_{\text {prior }}^{F}$ is zero, she is indifferent between these two options.

However, a third option is to attempt to gain additional information about the male's type before mating by engaging in extended courtship. This will be profitable if there is a prospect of obtaining a positive expected payoff higher than $E_{\text {prior }}^{F}$. In what follows we investigate equilibrium solutions with extended courtship.

\subsection{Cost of courtship}

In Sozou and Seymour (2005), the male signals his type to the female by offering a single costly gift. Here we consider courtship as a continuous process extended over

alternative formulation would be to assume that the male gets the same fitness payoff from mating whether he is a good or bad type, but that his cost of courting the female per unit time is greater if he is bad. Either assumption is equally acceptable in our model as the male's behaviour is determined by the ratio of his cost of courtship per unit time to his benefit from mating. Our results depend on this ratio being smaller if the male is a good type rather than a bad type, i.e. $\omega_{G}<\omega_{B}$ (see Table 1). 
time, and assume that this involves positive costs to both sexes. The female achieves a degree of screening by not being willing to mate immediately with the male, and the duration of a male's courtship effort constitutes a signal of his type.

Regardless of whether or not mating takes place, if the male expends time and effort courting the female he will incur a cost, and the female may also incur costs for time spent being courted by the male. In the human context, for example, suppose courtship involves a series of restaurant meals paid for by the male. Then the male must incur the cost of the meals and the cost of his time. The female gets a nutritional benefit from the meals, but must incur the cost of her time spent in restaurants. The cost of time is an opportunity cost. We assume that this opportunity cost exceeds the nutritional benefit to the female from each meal. Each individual meal may involve relatively small costs to the male and the female, but a number of meals over a long time period may add up to a significant total cost to both parties.

\subsection{The courtship process}

The game proceeds in continuous time. During courtship the male pays a constant $\operatorname{cost} x$ per unit time and the female pays a constant cost $\phi$ per unit time. We assume that $x$ and $\phi$ are environmentally determined variables. They are not strategic variables that either party can manipulate.

The game ends in one of three possible (independent) ways:

a) The female mates with the male.

b) The female quits the game without mating. 
c) The male quits the game and mating does not occur.

We assume behaviours are defined by constant probabilities per unit time or rates for each of the above processes. Thus, the female mates with a male at rate $\lambda$. The female quits without mating at rate $\theta$, and a male of type $K$ quits against a female at rate $q_{K}$. We then look for optimal values of these rates, where the male's rates are a best reply to those of the female and the female's rates are a best reply to those of a male who is good with probability $P_{f}$ and bad with probability $\left(1-P_{f}\right)$, to define a Nash equilibrium.

The above continuous time description of the game can be thought of as derived from a discrete-time game consisting of a sequence of rounds, each of short duration $\delta t$, by taking the limit $\delta t \rightarrow 0$. Each round of the discrete-time game will then consist of a sequence of moves. For example, in each round the male can decide to quit, with probability $q_{G} \delta t$ if his type is good, and $q_{B} \delta t$ if his type is bad. Similarly, the female quits without mating with probability $\theta \delta t$. If neither party quits, the female mates with probability $\lambda \delta t$, and the game then terminates. If none of these termination events occur, the game proceeds to the next round. Such a sequential interpretation is illustrated in Fig 1. However, it should be noted that the order in which events occur is irrelevant to first order in $\delta t$, and hence the continuous-time limit is compatible with many different sequential interpretations. 


\subsection{Duration and outcomes of the game}

Consider a courtship encounter between a female and a male of type $K$. As stated in section 2.5, the game ends in one of three ways: the male quits the game and mating does not occur; the female quits the game without mating; or the female mates with the male. The rates for these possible events are $q_{K}, \theta$ and $\lambda$ respectively. The total rate (i.e. probability per unit time) for the game ending is given by the sum of these rates, and hence is equal to $q_{K}+\theta+\lambda$. The duration of the game will therefore be exponentially distributed, with an expected duration given by $T_{K}=1 /\left(q_{K}+\theta+\lambda\right)$. The probability that the game ends with the male quitting is $q_{K} T_{K}$. The probability that the game ends with the female quitting without mating is $\theta T_{K}$. The probability that the game ends with mating taking place is $\lambda T_{K}$.

\subsection{Total payoffs from the game}

As long as the game continues, the male pays a cost $x$ per unit time. Thus, the expected total cost to the male is $x T_{K}$. The probability that the game ends with mating taking place is $\lambda T_{K}$, and in this case the male gains fitness payoff $M(K)$. Thus, the male's expected payoff is $E_{K}^{M}=M(K) \lambda T_{K}-x T_{K}$. From (1a), this yields:

$$
\begin{aligned}
& E_{G}^{M}=\frac{A_{m} \lambda-x}{q_{G}+\theta+\lambda}, \\
& E_{B}^{M}=\frac{D_{m} \lambda-x}{q_{B}+\theta+\lambda} .
\end{aligned}
$$


Similarly, let $E^{F}$ be the unconditional expected payoff to a female, allowing for the fact that she does not know the male's type. Then $E^{F}$ can be written as $\left[P_{f} \times\right.$ (expected payoff if the male is good $)]+\left[\left(1-P_{f}\right) \times(\right.$ expected payoff if the male is bad)]. That is:

$$
E^{F}=P_{f} \frac{A_{f} \lambda-\phi}{q_{G}+\theta+\lambda}-\left(1-P_{f}\right) \frac{C_{f} \lambda+\phi}{q_{B}+\theta+\lambda} .
$$

The first term in (5) corresponds to the female's prospect of being courted by a good male: she has a positive fitness term from the possibility that mating will occur, but a negative term from her expected cost of time spent in courtship. The second term corresponds to her prospect of being courted by a bad male: both the possibility of mating with such a male and the time spent in courtship constitute fitness costs for her. The payoff (5) therefore may be decomposed into components:

$$
E^{F}=E_{+}^{F}-E_{-}^{F},
$$

where:

$E_{+}^{F}=$ expected gain from mating $=\lambda\left\{P_{f} \frac{A_{f}}{q_{G}+\theta+\lambda}-\left(1-P_{f}\right) \frac{C_{f}}{q_{B}+\theta+\lambda}\right\}$,

$E_{-}^{F}=$ expected cost of courtship $=\phi\left\{P_{f} \frac{1}{q_{G}+\theta+\lambda}+\left(1-P_{f}\right) \frac{1}{q_{B}+\theta+\lambda}\right\}$. 
Note that $E_{-}^{F}$ is always non-negative, whereas $E_{+}^{F}$ can be positive, negative or zero.

\subsection{Evolutionary equilibria}

A male's strategy is a vector $\mathbf{s}_{M}=\left(q_{G}, q_{B}\right)$ of quitting rates, and a female's strategy is a vector $\mathbf{s}_{F}=(\theta, \lambda)$ of quitting and mating rates. A Nash Equilibrium (NE) is a pair of strategies, $\left(\mathbf{s}_{M}^{*}, \mathbf{s}_{F}^{*}\right)$, such that neither player can get a higher expected payoff by adopting a different strategy, i.e.:

$E^{F}\left(\mathbf{s}_{M}^{*}, \mathbf{s}_{F}^{*}\right) \geq E^{F}\left(\mathbf{s}_{M}^{*}, \mathbf{s}_{F}\right) \quad$ for all $\mathbf{s}_{F}$, and

$E_{K}^{M}\left(\mathbf{s}_{M}^{*}, \mathbf{s}_{F}^{*}\right) \geq E_{K}^{M}\left(\mathbf{s}_{M}, \mathbf{s}_{F}^{*}\right) \quad$ for $K=G$ or $B$, and all $\mathbf{s}_{M}$.

That is, each strategy $\mathbf{s}_{M}^{*}$ and $\mathbf{s}_{F}^{*}$ is a best reply to the other.

We look for mating solutions with extended courtship, i.e. Nash equilibria in which mating is not immediate and occurs with probability greater than zero. We analyse the game in a series of steps, progressively making deductions about the nature of any such solutions of the game. Evolutionary stability of equilibria is discussed in section 3.6 and in Appendix A.

\section{Analysis}

\subsection{Notation for detailed analysis}

We define the following cost-benefit ratios (each having the dimensions of time ${ }^{-1}$ ): 


$$
\omega_{G}=\frac{x}{A_{m}}, \quad \omega_{B}=\frac{x}{D_{m}}, \quad \sigma_{G}=\frac{\phi}{A_{f}}, \quad \sigma_{B}=\frac{\phi}{C_{f}} .
$$

Table 1 summarises the notation used in this paper.

Note that (2a) implies that $0<\omega_{G}<\omega_{B}$. In other words, the ratio of the male's cost per unit time of courting the female to his gain from mating is smaller if his type is good.

Table 1. Major symbolic notation used in this paper with the associated meaning.

\begin{tabular}{|c|l|c|l|}
\hline \multicolumn{2}{|c|}{ Female } & \multicolumn{2}{c|}{ Male } \\
\hline Symbol & \multicolumn{1}{|c|}{ Meaning } & Meaning \\
\hline- & \multicolumn{1}{|c|}{$P_{f}$} & $\begin{array}{l}\text { Prior probability that male type is } \\
\text { "good" }\end{array}$ \\
\hline$A_{f}$ & $\begin{array}{l}\text { Fitness gain to female from } \\
\text { mating with a good male }\end{array}$ & $A_{m}$ & Fitness gain to a good male from \\
& Fitness cost to female from & $D_{m}$ & Fitness gain to a bad male from mating \\
\hline$C_{f}$ & mating with a bad male & & \\
\hline$E^{F}$ & Total expected payoff to a female & $E_{K}^{M}$ & Total expected payoff to a male of \\
& from courtship & & type K (= $G$ or $B)$ from courting \\
\hline$\phi$ & Cost of courtship per unit time & $x$ & Cost of courtship per unit time \\
\hline$\theta$ & Rate of quitting against male & $q_{K}$ & Rate of quitting by male of type $K(=$ \\
& & & $G$ or $B)$ \\
\hline$\lambda$ & Rate of mating with courting & $\hat{q}_{B}$ & Lower bound for $q_{B}$ to define a Nash \\
& male & & equilibrium (section 3.5) \\
\hline$\sigma_{L}$ & $\phi / A_{f}(L=G) ; \phi / C_{f}(L=B)$ & $\omega_{K}$ & $x / A_{m} \quad(K=G) ; x / D_{m} \quad(K=B)$ \\
\hline
\end{tabular}




\begin{tabular}{|l|l|l|l|}
\hline $\begin{array}{l}\text { Ratio of female's cost per unit } \\
\text { time for participating in the } \\
\text { courtship process to her gain or } \\
\text { loss from mating with a male of } \\
\text { type } L\end{array}$ & & Ratio of male's cost per unit time for \\
courting to his gain from mating, \\
where $K$ represents the male's type
\end{tabular}

Using the notation of (8), the male expected payoffs $(4 a, b)$ can be expressed as:

$$
E_{G}^{M}=x \frac{\lambda-\omega_{G}}{\omega_{G}\left(q_{G}+\theta+\lambda\right)}, \quad E_{B}^{M}=x \frac{\lambda-\omega_{B}}{\omega_{B}\left(q_{B}+\theta+\lambda\right)}
$$

Notice that the male gets a positive payoff if and only if the female's mating rate $\lambda$ is higher than the ratio of the male's courtship cost per unit time to his gain from mating ( $\omega_{G}$ if he is a good type, or $\omega_{B}$ if he is a bad type).

The female's expected payoff (5) may be expressed as:

$$
E^{F}=\phi\left\{P_{f} \frac{\lambda-\sigma_{G}}{\sigma_{G}\left(q_{G}+\theta+\lambda\right)}-\left(1-P_{f}\right) \frac{\lambda+\sigma_{B}}{\sigma_{B}\left(q_{B}+\theta+\lambda\right)}\right\} .
$$

Notice from the first term inside the curly brackets in (10) that, even if the female is sure that the male's type is good (i.e. if $P_{f} \rightarrow 1$ ), the female's mating rate $\lambda$ must be greater than $\sigma_{G}$ for her to get a positive expected fitness benefit from the game. If it is less, then her expected cost of time spent on courtship will be greater than her gain 
from mating (unless she quits immediately with $\theta=\infty$, in which case she will spend no time on courtship and mating will not occur).

A summary of the main findings of the analysis that follows is given at the end of this section, in section 3.8 .

\subsection{The female never quits}

If the female is to obtain a positive total expected payoff $E^{F}=E_{+}^{F}-E_{-}^{F}$ (see equation (6a)), the expected fitness cost of the time she spends being courted, $E_{-}^{F}$, must be less than her expected fitness gain from mating $E_{+}^{F}$. This requires that she must mate with a sufficiently high rate: specifically $\lambda>\sigma_{G}$ (see (10)). Otherwise her expected payoff would be negative, and she would do better by quitting immediately (i.e. $\theta=\infty$ ) to obtain a zero payoff. Thus, an equilibrium with mating requires that $\lambda>\sigma_{G}$ and $\theta$ finite.

We now show that a female strategy with $\theta>0$ cannot be optimal. Consider such a strategy, $(\theta, \lambda)$, with positive $\theta$ and $\lambda$ finite. If this is not worse for the female than quitting immediately then it must give a non-negative expected payoff, i.e.

$E^{F}(\theta, \lambda)=E_{+}^{F}(\theta, \lambda)-E_{-}^{F}(\theta, \lambda) \geq 0$. As she has a positive expected cost of courtship $E_{-}^{F}(\theta, \lambda)$, it follows that her expected gain from mating $E_{+}^{F}(\theta, \lambda)$ must also be positive. Now consider the alternative female strategy $(0, \theta+\lambda)$; this has the same expected duration of courtship, but the female never quits and the game is more likely to end in mating. From (6b), the alternative strategy has the same expected cost of 
courtship, i.e. $E_{-}^{F}(0, \theta+\lambda)=E_{-}^{F}(\theta, \lambda)$. However, from (6a), the female's expected gain from mating is now $E_{+}^{F}(0, \theta+\lambda)=(\theta+\lambda) / \lambda \times E_{+}^{F}(\theta, \lambda)$. Thus, since $E_{+}^{F}(\theta, \lambda)$ is positive and $\theta>0$, it follows that $E_{+}^{F}(0, \theta+\lambda)>E_{+}^{F}(\theta, \lambda)$, and hence for total expected payoffs, that $E^{F}(0, \theta+\lambda)>E^{F}(\theta, \lambda)$. Therefore the alternative strategy gives the female a higher total expected payoff.

This shows that any female strategy $(\theta, \lambda)$ that involves quitting with positive probability (i.e. with $\theta>0$ and $\lambda$ finite) and that gives a positive expected payoff will be less fit than a strategy that involves never quitting.

If the female mates immediately (i.e. $\lambda=\infty$ ), then she receives expected payoff $E_{\text {prior }}^{F}$ given by (3), which is independent of $\theta$. Hence, any finite value of $\theta$ is irrelevant to her expected payoff if she mates immediately. There is therefore no loss of generality in assuming that $\theta=0$ in this case also. Hence, in any equilibrium with mating, we can assume that the female never quits (i.e. $\theta=0$ ).

Now observe from (10) that the condition $E^{F}(0, \lambda)>0$ for a positive expected payoff for the female if she never quits may be expressed in the form:

$$
P_{f}>\frac{\sigma_{G}\left(\lambda+\sigma_{B}\right)\left(q_{G}+\lambda\right)}{\sigma_{G}\left(\lambda+\sigma_{B}\right)\left(q_{G}+\lambda\right)+\sigma_{B}\left(\lambda-\sigma_{G}\right)\left(q_{B}+\lambda\right)} .
$$




\subsection{A good male never quits}

From the result in section 3.2 that, if she does not quit immediately, the female's optimal quitting strategy is $\theta=0$, it follows that the male and female expected payoffs $(9,10)$ reduce to:

$$
\begin{aligned}
& E_{G}^{M}=x \frac{\lambda-\omega_{G}}{\omega_{G}\left(q_{G}+\lambda\right)}, \quad E_{B}^{M}=x \frac{\lambda-\omega_{B}}{\omega_{B}\left(q_{B}+\lambda\right)}, \\
& E^{F}=\phi\left\{P_{f} \frac{\lambda-\sigma_{G}}{\sigma_{G}\left(q_{G}+\lambda\right)}-\left(1-P_{f}\right) \frac{\lambda+\sigma_{B}}{\sigma_{B}\left(q_{B}+\lambda\right)}\right\} .
\end{aligned}
$$

The female's strategy is now completely determined by her choice of mating frequency $\lambda$. The male's strategy is determined by his quitting vector $\mathbf{s}_{M}=\left(q_{G}, q_{B}\right)$. Table 2 shows the male's best reply (BR) to various values of $\lambda$, and in turn the female's best reply $\lambda$ to each quitting strategy $\mathbf{s}_{M}$.

Table 2. Five cases (column 1), with possible ranges of the female mating rate $\lambda$ given in column 2 . The third and fourth columns give the best reply (BR) male quitting strategies to any $\lambda$ in the given range. The fifth column gives the female's best reply $\lambda$ to the male quitting strategy defined in the third and fourth columns.

\begin{tabular}{|c|c|c|c|c|}
\hline Case & $\lambda$ range & BR $q_{G}$ & BR $q_{B}$ & BR $\lambda$ \\
\hline 1 & $\lambda<\omega_{G}<\omega_{B}$ & $\infty$ & $\infty$ & undetermined \\
\hline 2 & $\lambda=\omega_{G}<\omega_{B}$ & undetermined & $\infty$ & $\infty$ \\
\hline 3 & $\omega_{G}<\lambda<\omega_{B}$ & 0 & $\infty$ & $\infty$ \\
\hline 4 & $\omega_{G}<\lambda=\omega_{B}$ & 0 & undetermined & undetermined \\
\hline
\end{tabular}




\begin{tabular}{|c|c|c|c|c|}
\hline 5 & $\omega_{G}<\omega_{B}<\lambda$ & 0 & 0 & $\infty$ \\
\hline
\end{tabular}

From the male's point of view, the game is very much like a war of attrition (Maynard Smith, 1974): he is waiting for a resource (mating) and faces a constant hazard rate of $\lambda$ for gaining the resource. The ratio of his cost of courtship per unit time to the value of the resource to him is $\omega_{G}$ (if he is good) or $\omega_{B}$ (if he is bad). If $\lambda$ is less than this ratio he should quit immediately. If $\lambda$ is greater than this ratio he should wait indefinitely without quitting. If $\lambda$ is exactly equal to this ratio then all values of his quitting rate are equal best replies, giving him an expected payoff of zero.

Cases 2 and 3 in Table 2 cannot define Nash equilibria since the values of $\lambda$ in the second and fifth columns are incompatible. For values of $\lambda$ in case 1 , the male cannot obtain a positive payoff from any female, and so the best he can do is to quit immediately to obtain zero payoff. In this case there is no courtship. In contrast, values of $\lambda$ in case 5 are so large that the male's best strategy is never to quit. The female's best reply is then to mate immediately, since she cannot avoid mating with a bad male by waiting for him to quit first.

It follows that the only possible Nash equilibria in which mating occurs arise from cases 4 and 5, with extended courtship being possible only in case 4 . In both these cases $q_{G}=0$, i.e. a good male does not quit. 


\subsection{Best reply strategies}

The female's best reply choice of $\lambda$ to a bad male's quitting strategy $q_{B}$ is obtained by maximizing $E^{F}(\lambda)$ given by (13). To find this value, consider

$\frac{1}{\varphi} \frac{d E^{F}}{d \lambda}=P_{f} \frac{1}{\lambda^{2}}-\left(1-P_{f}\right) \frac{q_{B}-\sigma_{B}}{\sigma_{B}\left(q_{B}+\lambda\right)^{2}}$.

There are several cases.

If $q_{B} \leq \sigma_{B}$ then $d E^{F} / d \lambda$ is always positive, and $E^{F}(\lambda)$ is monotonically increasing in $\lambda$. Hence $E^{F}(\lambda)$ is maximized at $\lambda=\infty$. From (13), (8) and (3), $E^{F}(\infty)=E_{\text {pror }}^{F}$, and it follows that the female should mate immediately if $E_{\text {prior }}^{F}>0$, but should quit immediately if $E_{\text {prior }}^{F}<0$ (to obtain payoff 0 ). If $E_{\text {prior }}^{F}=0$, the female is indifferent between these two options.

If $q_{B}>\sigma_{B}$, then $d E^{F} / d \lambda \geq 0$ if and only if:

$1+\frac{q_{B}}{\lambda} \geq \sqrt{\frac{\left(1-P_{f}\right)\left(q_{B}-\sigma_{B}\right)}{P_{f} \sigma_{B}}}$.

This holds for any $\lambda \geq 0$ when the right-hand-side is $\leq 1$. That is, when

$q_{B} \leq \frac{\sigma_{B}}{1-P_{f}}$. 
Again, the female's best reply is to mate immediately $(\lambda=\infty)$ if $E_{p r i o r}^{F}>0$, but to quit immediately if $E_{p r i o r}^{F}<0$. Clearly condition (15) includes the case $q_{B} \leq \sigma_{B}$.

Finally, if

$$
q_{B}>\frac{\sigma_{B}}{1-P_{f}}
$$

then (14) yields a unique, finite value $\lambda=\lambda^{*}\left(q_{B}\right)$ at which $E^{F}$ is maximized. This is given by:

$$
\lambda^{*}\left(q_{B}\right)=\frac{q_{B} \sqrt{P_{f} \sigma_{B}}}{\sqrt{\left(1-P_{f}\right)\left(q_{B}-\sigma_{B}\right)}-\sqrt{P_{f} \sigma_{B}}} .
$$

The denominator of $\lambda^{*}\left(q_{B}\right)$ is positive when (16) holds, and clearly $\lambda^{*}\left(q_{B}\right) \rightarrow \infty$ as $q_{B} \rightarrow \sigma_{B} /\left(1-P_{f}\right)$. At the other extreme, $\lambda^{*}\left(q_{B}\right) \rightarrow \infty$ as $q_{B} \rightarrow \infty$. It is easy to show that $\lambda^{*}\left(q_{B}\right)$ has a unique minimum at $q_{B}=q_{*}$, where $q_{*}=2 \sigma_{B} /\left(1-\sqrt{P_{f}}\right)$, and that $\lambda_{\min }^{*}=\lambda^{*}\left(q_{*}\right)=2 \sigma_{B} \sqrt{P_{f}} /\left(1-\sqrt{P_{f}}\right)$. From case 4 of Table 2 , the requirement that $q_{B}$ defines a Nash equilibrium is that $q_{B}$ is a solution of $\lambda^{*}\left(q_{B}\right)=\omega_{B}$, and it follows from the above analysis that this will be the case if and only if $\lambda_{\min }^{*} \leq \omega_{B}$, which gives:

$$
P_{f} \leq\left(\frac{\omega_{B}}{2 \sigma_{B}+\omega_{B}}\right)^{2} .
$$


Further, if this inequality is strict, then there are exactly two such solutions for $q_{B}$. We denote these by $q_{0}^{*}$ and $q_{1}^{*}$ with $q_{0}^{*} \leq q_{*} \leq q_{1}^{*}$ (see Fig 2). Clearly, (18) will always hold if $\omega_{B}$ is sufficiently large, and from the definition (8) this holds if either the courtship cost $x$ is large, or the fitness gain $D_{m}$ to a bad male from mating is small. On the other hand, if $\omega_{B}$ is sufficiently small, then (18) is not satisfied, and in this case there can be no equilibrium solutions with extended courtship. The female's best response is then either to mate immediately (if she gets a positive payoff) or to quit immediately (otherwise). This case arises if either the courtship cost $x$ is small or the fitness gain $D_{m}$ is large.

\section{FIG 2 HERE}

\subsection{The condition for the female to get a positive payoff}

When (16) holds, so that there may be extended courtship equilibria, it remains to consider whether the condition (11) for a female to obtain a positive expected payoff also holds. Substituting the values $q_{G}=0, \lambda=\omega_{B}$ (from case 4 of Table 2) we find that (11) holds if and only if $\omega_{B}>\sigma_{G}$ and $q_{B}>\hat{q}_{B}$, where:

$$
\hat{q}_{B}=\omega_{B}\left\{\frac{\left(1-P_{f}\right) \sigma_{G}\left(\omega_{B}+\sigma_{B}\right)}{P_{f} \sigma_{B}\left(\omega_{B}-\sigma_{G}\right)}-1\right\} .
$$


It is possible to obtain $\hat{q}_{B}<q_{0}^{*}<q_{1}^{*}$, in which case both $q_{0}^{*}$ and $q_{1}^{*}$ are Nash equilibria (and from section 3.6 below, $q_{1}^{*}$ is then evolutionarily stable). It is also possible to obtain $q_{0}^{*}<q_{1}^{*}<\hat{q}_{B}$, in which case there are no Nash equilibria. In this case, the female cannot obtain a positive payoff by mating (either immediately or otherwise), and she should quit immediately. These cases are illustrated in Fig 2.

\subsection{Evolutionary stability}

As we have seen in the preceding analysis, there are two possible Nash equilibria determined by bad male quitting strategies $q_{0}^{*}, q_{1}^{*}$. In both cases the female's mating strategy is $\lambda=\omega_{B}$. We have also seen (Fig 2) that often $q_{0}^{*}$ is not compatible with (11) (i.e. $q_{0}^{*}<\hat{q}_{B}$ ), and sometimes neither $q_{0}^{*}$ nor $q_{1}^{*}$ is compatible with (11). As noted above, if (11) is not satisfied, there cannot be a Nash equilibrium with mating as females would always do best to quit immediately without mating. However, sometimes both strategies are compatible with (11) (i.e. $\hat{q}_{B}<q_{0}^{*}<q_{1}^{*}-$ see Fig 2). In this section we show that if $q_{1}^{*}$ is compatible with (11), and therefore an equilibrium solution, then it is evolutionarily stable, but if $q_{0}^{*}$ is compatible with (11) it is evolutionarily unstable.

Fig 3a shows the female's best reply curve $\lambda=\lambda^{*}\left(q_{B}\right)$, and Fig $3 b$ shows the male's best reply curve $q_{B}=q_{B}^{*}(\lambda)$. First consider the equilibrium $\left(q_{B}, \lambda\right)=\left(q_{0}^{*}, \omega_{B}\right)$. Suppose a small perturbation decreases $q_{B}$ below $q_{0}^{*}$. Then the female's best reply satisfies $\lambda^{*}\left(q_{B}\right)>\omega_{B}$ (Fig 3a). Thus, there will be selection pressure to increase $\lambda$ 
above $\omega_{B}$. But then the male's best reply is $q_{B}^{*}(\lambda)=0$ (Fig $\left.3 b\right)$. It follows that there will be selection pressure to decrease $q_{B}$ still further, and hence the equilibrium $\left(q_{0}^{*}, \omega_{B}\right)$ is unstable. Similarly, a small perturbation in $q_{B}$ above $q_{0}^{*}$ will be amplified by selection.

The reverse argument applies to the equilibrium $\left(q_{1}^{*}, \omega_{B}\right)$, so that small perturbations in $q_{B}$, either above or below $q_{1}^{*}$, will die out under selection pressure. If a small perturbation increases $\lambda$ above $\omega_{B}$, then the male's best reply is $q_{B}^{*}(\lambda)=0$. There will therefore be selection pressure to decrease $q_{B}$ below $q_{1}^{*}$. But then the female's best reply is $\lambda^{*}\left(q_{B}\right)<\omega_{B}$, so there will be selection pressure to reverse her excursion above $\lambda=\omega_{B}$. A similar argument applies to a small perturbation that decreases $\lambda$ below $\omega_{B}$.

\section{FIG 3 HERE}

More generally, a perturbation away from equilibrium has the form $\left(q_{1}^{*}, \omega_{B}\right) \rightarrow\left(q_{1}^{*}+r, \omega_{B}+s\right)$ with $r$ and $s$ small. Assuming an evolutionary dynamics which tends to move $q_{B}$ and $\lambda$ in the direction of best replies, the possible evolutionary trajectories are illustrated in Fig 4. However, this picture is inconclusive, and suggests that there could be cycles around equilibrium. In Appendix A we consider stability with respect to a particular class of best reply evolutionary dynamics, and show that $\left(q_{1}^{*}, \omega_{B}\right)$ is in fact locally asymptotically stable with respect to any dynamics in this class. 


\section{FIG 4 HERE}

\subsection{Equilibrium outcomes}

We have shown that there are three possible equilibrium behaviours for the female: a) quit immediately, in which case no courtship or mating occurs; b) mate immediately; c) engage in extended courtship. It is optimal for the female to quit immediately, giving her a payoff of zero, if she cannot obtain a positive payoff from behaviour b) or behaviour c). Conversely, if her payoff $E_{\text {prior }}^{F}$ (equation (3)) from immediate mating is positive then this will be better than quitting immediately. But if she can get a higher payoff from engaging in extended courtship, then her best strategy is to engage in extended courtship rather than to mate immediately.

Using equation (3) and the notation (8), $E_{\text {prior }}^{F}$ is positive if and only if $P_{f}>P_{f}^{*}$, where:

$$
P_{f}^{*}=\frac{\sigma_{G}}{\sigma_{G}+\sigma_{B}} .
$$

As discussed in section 3.4, the Nash equilibrium with extended courtship, $\left(q_{B}, \lambda\right)=\left(q_{1}^{*}, \omega_{B}\right)$, can exist only if $P_{f} \leq \alpha^{2}$, where $\alpha=\omega_{B} /\left(2 \sigma_{B}+\omega_{B}\right)$ (see (18)), and the female can obtain a positive payoff from this behaviour only if $q_{1}^{*}>\hat{q}_{B}$, given by (19). In this case, the female's expected payoff from extended courtship is necessarily larger than $E_{\text {prior }}^{F}$. 
In this section, we regard the female cost-benefit parameters $\sigma_{G}$ and $\sigma_{B}$ as fixed, and consider the possible female equilibrium behaviours as functions of the male parameters $P_{f}$, the prior probability that a male is good, and $\omega_{B}$, a bad male's costbenefit ratio.

From this perspective, $P_{f}^{*}$ (equation (20)) is fixed and $\alpha$ is a function of $\omega_{B}$. Thus, the region in the $\left(P_{f}, \omega_{B}\right)$-plane in which $E_{\text {prior }}^{F}$ is negative is separated from the region in which it is positive by the line $P_{f}=P_{f}^{*}$ (Fig 5). Similarly, the threshold in the $\left(P_{f}, \omega_{B}\right)$-plane separating the region in which $q_{1}^{*}$ exists from the region in which it does not is determined by the relation $P_{f}=\alpha^{2}$. That is, by the curve $\omega_{B}=2 \sigma_{B} \sqrt{P_{f}} /\left(1-\sqrt{P_{f}}\right)$.

The equilibrium quitting rate for a bad male, $q_{1}^{*}$, when it exists, may or may not give the female a positive payoff. By definition, $q_{1}^{*}$ is the larger root of the equation $\lambda^{*}\left(q_{B}\right)=\omega_{B}$, in which $\lambda^{*}\left(q_{B}\right)$, given by (17), is a function of $P_{f}$. Thus, $q_{1}^{*}$ is a function of $P_{f}$ and $\omega_{B}$. Clearly, the threshold $\hat{q}_{B}$, given by (19), is also a function of $P_{f}$ and $\omega_{B}$. It follows that the region in the $\left(P_{f}, \omega_{B}\right)$-plane in which there is a Nash equilibrium with extended courtship is defined by the relations $q_{1}^{*}>\hat{q}_{B}$ and $P_{f}<\alpha^{2}$. In Appendix B we show that these conditions define a curve $\omega_{B}=f\left(P_{f}\right)$, with $f\left(P_{f}\right)$ a continuous, monotonically increasing function, such that extended courtship 
is an ESS outcome if and only if $\omega_{B}>f\left(P_{f}\right)$. Further, $f\left(P_{f}\right) \rightarrow \infty$ as $P_{f} \rightarrow 1$, and $f(0)=\hat{\omega}_{B}$, where

$\hat{\omega}_{B}=\sigma_{G}+\sqrt{\sigma_{G}\left(\sigma_{G}+\sigma_{B}\right)}$.

In particular, there can be no ESS with extended courtship when $\omega_{B}<\hat{\omega}_{B}$.

The various possible outcomes, for fixed values of $\sigma_{G}$ and $\sigma_{B}$, are illustrated in Fig 5 as regions in the $\left(P_{f}, \omega_{B}\right)$-plane. There are four major regions. In Region $\mathrm{A}$, extended courtship is the unique ESS; in Region B, the female cannot obtain a positive payoff, and therefore quits immediately, while in Region $\mathrm{C}$ there are sufficiently many good males in the population that she can obtain a maximum, positive payoff from mating immediately. In Region D, the female can obtain a positive payoff either from mating immediately, or from engaging in extended courtship.

However, in Region D, the female payoff from extended courtship is greater than that from mating immediately (section 3.4). In this case, if the population is in the extended courtship equilibrium, then a female's best strategy is also to engage in extended courtship. For males this leads to the apparent paradox that, even though there may be an alternative equilibrium in which all males mate immediately and there are no costs of extended courtship, once the extended courtship equilibrium is established, any male who unilaterally decides not to engage in extended courtship will not get to mate. 
At the other extreme, when $P_{f} \rightarrow 0$, there are very few good males in the population. If the cost-benefit ratio $\omega_{B}$ for a bad male is sufficiently small, the female will quit immediately and mating will not occur (Region B). However, if $\omega_{B}$ is sufficiently large (i.e. $\omega_{B}>\hat{\omega}_{B}$; Region A) an equilibrium with courtship exists, and it is shown in Appendix B that $q_{1}^{*} \rightarrow \infty$ as $P_{f} \rightarrow 0$. Thus, bad males quit increasingly quickly at equilibrium as $P_{f}$ becomes progressively smaller. As $P_{f} \rightarrow 0$, the equilibrium has the following characteristics:

- The proportion of courtship encounters in which the male is good tends towards zero, i.e. the probability that a random male is bad tends towards 1 ;

- The probability that an encounter between a female and bad male will lead to mating tends towards zero, and the overall probability that an encounter between a female and a random male will lead to mating also tends towards zero;

- The expected duration of courtship in an encounter between a female and a bad male, and the overall expected duration of courtship, both tend towards zero.

Note that as $P_{f} \rightarrow 0$ within region A, the bad male's quitting rate $q_{1}^{*}$ is the only behavioural variable that changes at equilibrium. A female will continue to delay mating with a mean waiting time before mating of $1 / \lambda=1 / \omega_{B}$. Thus, it becomes increasingly probable that a female will screen out (or "outwait") a bad male. In contrast, a good male will continue to wait indefinitely. It follows that an encounter between a female and a good male will continue to lead to mating, with a mean courtship time for such an encounter of $1 / \omega_{B}$. However, encounters between a female and a good male will be increasingly rare as $P_{f} \rightarrow 0$. 
Of particular note is the fact that, for some values of $\omega_{B}$, the range of $P_{f}$ values inside Region A in which extended courtship is an equilibrium outcome, is separated from the range of $P_{f}$ values in region $\mathrm{C}$ in which immediate mating is the equilibrium outcome, by a range of $P_{f}$ values in region B in which no mating occurs. Thus, as more good males are introduced into the population ( $P_{f}$ is increased), the equilibrium outcome can first change from one in which extended courtship is possible to one in which no mating occurs, before reaching a state in which such a high proportion of males are good that immediate mating becomes optimal for the female.

To understand this counter-intuitive phenomenon, consider a courtship equilibrium $\left(\lambda=\omega_{B}, q_{\mathrm{B}}=q_{1}^{*}\right)$. In the vicinity of the equilibrium, the female's best response $\lambda^{*}\left(q_{B}\right)$ is an increasing function of $q_{B}$ (Fig 3a). As $P_{f}$ increases, the female's bestresponse curve, $\lambda^{*}\left(q_{B}\right)$, moves upwards - see (17). It follows that $q_{1}^{*}$ decreases. Hence, a bad male's equilibrium expected waiting time before quitting, $1 / q_{1}^{*}$, increases. The female's equilibrium expected waiting time remains the same, $1 / \omega_{B}$. This means that a female will screen out (or "outwait") a smaller proportion of bad males, so that she now has a greater risk that a courtship encounter with a bad male will lead to mating. Reducing $\lambda$ to increase her expected waiting time would increase her chance of screening out a bad male but would not benefit her overall because she would have to pay an increased expected cost of waiting time. The result is that when $q_{1}^{*}$ becomes too low the female cannot get a positive expected payoff from extended 
courtship, and since she cannot obtain a positive payoff from mating immediately (because $P_{f}<P_{f}^{*}$ ), her best option is to quit immediately.

A transition from region $\mathrm{A}$ to region $\mathrm{B}$ as $P_{f}$ is increased can be understood from the perspective of selection pressure and evolutionary dynamics. Suppose that $P_{f}$ increases, i.e. more good males are introduced to the population, with $q_{B}$ initially remaining at the same value as before. Then the best response value of $\lambda$ increases: there will be selection for females to reduce the delay before mating. This in turn leads to a lower best response value of $q_{B}$, i.e. there will be selection for a lower quitting rate for bad males. Eventually a new equilibrium will be established at which $\lambda$ has the same value of $\lambda=\omega_{B}$ as before, but the equilibrium bad male quitting rate

$q_{1}^{*}$ is now lower. As $P_{f}$ is increased, there comes a point where $q_{1}^{*}$ is too low to give the female a positive payoff; at this point courtship collapses. From a female perspective, as $P_{f}$ increases the benefit of a higher proportion of good males is outweighed by the lower equilibrium quitting rate of bad males.

\section{FIG 5 HERE}

\subsection{Summary of results}

We have found conditions for the existence of Nash equilibria in which there is extended courtship resulting in mating with positive probability. The characteristics of such equilibria are summarized as follows: 
- The female obtains a positive expected payoff and never quits $(\theta=0$; section 3.2).

- A good male obtains a positive expected payoff and never quits $\left(q_{G}=0\right.$; section 3.3).

- An equilibrium is therefore defined by $\left(q_{B}, \lambda\right)$, where $\lambda$ is the female's mating rate and $q_{B}$ is a bad male's quitting rate.

- At an equilibrium with extended courtship, the female's mating strategy is $\lambda=\omega_{B}($ section 3.3$)$.

- There are 0,1 or 2 possible male Nash equilibrium quitting strategies, $q_{B}=q_{0}^{*}$ or $q_{1}^{*}$, with $q_{1}^{*} \geq q_{0}^{*}>\sigma_{B} /\left(1-P_{f}\right)$ (section 3.4 and Fig 2$)$.

- There is a threshold value $\hat{q}_{B}$ such that $q_{i}^{*}$ defines a Nash equilibrium if and only if $q_{i}^{*}>\hat{q}_{B}$, this being the condition for the female to obtain a positive expected payoff (section 3.5 and Fig 2).

- If the Nash equilibrium $\left(q_{B}, \lambda\right)=\left(q_{0}^{*}, \omega_{B}\right)$ exists, then it is evolutionarily unstable (section 3.6).

- If the Nash equilibrium $\left(q_{B}, \lambda\right)=\left(q_{1}^{*}, \omega_{B}\right)$ exists, then it is evolutionarily stable with respect to a class of best-response evolutionary dynamics (section 3.6)

- There is a threshold value $\hat{\omega}_{B}$, depending on only the female cost-benefit ratios $\sigma_{G}$ and $\sigma_{B}$, such that there is a non-empty range of $P_{f}$ containing equilibria with extended courtship only for $\omega_{B}>\hat{\omega}_{B}$ (section 3.7 and Fig 5).

- There is a threshold value $P_{f}^{*}$, depending only on $\sigma_{G}$ and $\sigma_{B}$, such that the female obtains a positive payoff from mating immediately if and only if $P_{f}>P_{f}^{*}$ 
- For $\omega_{B}<\hat{\omega}_{B}$ the female's optimal strategy is to quit immediately if $P_{f}<P_{f}^{*}$, and to mate immediately if $P_{f}>P_{f}^{*}$ (section 3.7 and Fig 5).

We conclude that there is at most one equilibrium with extended courtship that is evolutionarily stable. For any value of $P_{f}$ in the range $0 \leq P_{f}<1$, an equilibrium is guaranteed if a bad male's cost-benefit ratio $\omega_{B}$ is sufficiently large.

\section{Discussion}

In this study we have modelled courtship as a waiting game over continuous time. The game ends with one or other party quitting (to pursue outside options), or with mating taking place. Strategies are defined by fixed probabilities per unit time, or rates, for each of the possible moves which end the game. In contrast to Sozou and Seymour (2005), who considered the type of gift appropriate in courtship, in the present study we make the assumption that extended courtship involves costs to both the male and the female, and we are concerned with understanding the role of the duration of courtship effort as a signal. During courtship, both the male and the female pay participation costs per unit time at fixed rates. A courtship process involving a sequence of small gifts fits the assumptions of this model as long as the gifts are "costly but worthless", i.e. the time-cost to the female in receiving the gifts exceeds her intrinsic (i.e. non-informational) benefit from the gifts. But the model is more general than this in that it considers a courtship process which involves participation costs but need not involve gifts as such. 
A key feature of the model is asymmetric information arising from a binary variable, not completely observable by the female. The female will get a positive payoff from mating only if the male is a "good" male, with respect to his genetic quality, or ability or intention to provide paternal care. If the female mates with a "bad" male, i.e. one who is low quality or will not provide paternal care, she will get a negative payoff from mating with him.

We have found evolutionarily stable behaviour in which mating occurs after extended courtship. This has the following characteristic: a "bad" male should quit the courtship process at a certain positive rate, whereas a "good" male should persist for longer (indefinitely for the specific assumptions in our model). To the male, the game has some similarity to a war of attrition, with the opportunity to mate with the female constituting the resource for which he is waiting. A "good" male has a higher ratio of fitness benefit from mating to fitness cost per unit time of courting than a "bad" male.

From the female's point of view, the strategic problem that she faces is one of decision-making under uncertainty (Dall et al., 2005). Whereas the model of Sozou and Seymour (2005) leads to an equilibrium outcome in which the female never mates with a "bad" male, in real mating systems females may sometimes mate with the wrong male. This could be because of random errors in a female's assessment of male quality (Luttbeg, 1996) or limits on the processing capacity of her neural system (Krakauer and Johnstone, 1995).

The extended courtship equilibria in the present study also do not completely eliminate the risk of a female mating with a "bad" male. The female's strategy is a 
compromise solution in the face of a trade-off between the costs of mating too quickly (an increased risk of mating with a "bad" male) and the time-cost of delay. The female's cost of delay can be interpreted as a cost of acquiring information.

Bad males quit at a finite rate; they do not quit immediately. This means that, whilst the courtship cost incurred by a bad male is on average less than that incurred by a good male, it is nevertheless positive. It is therefore necessary for bad males to sometimes succeed in mating in order for an extended courtship equilibrium to be sustained. Appendix $\mathrm{C}$ extends the analysis to an arbitrary number of discrete male types of increasing "goodness". The extended model behaves in all essential respects like the binary model: the worst type of male that courts the female constitutes the "bad" male type and has a finite quitting rate, whilst all better male types never quit and collectively constituting the equivalent of the "good" male type.

In both the binary and extended version of the model, equilibrium behaviour is qualitatively determined by the cost-benefit ratio $\omega_{B}$ of a bad male, and the probability $P_{f}$ that a (random) courtship encounter will be with a good male. A good male always has a lower cost-benefit ratio. If $\omega_{B}$ is below a threshold (determined by the female cost-benefit ratios), then the female's only possible equilibrium behaviour is either to mate immediately if $P_{f}$ is high enough for her expected payoff from immediate mating to be positive, or to quit immediately if $P_{f}$ is too low and her expected payoff is negative. However, as $\omega_{B}$ increases above this threshold, there is an increasing range of values of $P_{f}$ for which extended courtship is evolutionarily stable. For given $\omega_{B}$, this range of $P_{f}$ extends from 0 to some threshold. For $P_{f}$ 
beyond this threshold (i.e. as the proportion of good males increases), extended courtship ceases to be a viable equilibrium, and female behaviour will revert to either quitting immediately or mating immediately (section 3.7 and Fig 5). This leads to the counterintuitive possibility that, for some ranges of parameters, an evolutionarily stable extended courtship equilibrium with mating is possible for low $P_{f}$, and immediate mating will occur for high $P_{f}$, but for intermediate values of $P_{f}$ no mating will occur. In other words, as $P_{f}$ increases mating will break down when there are too many good males in the population to enable an extended courtship equilibrium, but not enough to make immediate mating profitable for the female.

For the cost-benefit ratio $\omega_{B}$, and the proportion of good males $P_{f}$ both sufficiently large, it is possible for a female to obtain a positive expected payoff from both extended-courtship and mating immediately. However, the payoff she obtains in a population in an extended courtship equilibrium is always strictly higher than that obtained from mating immediately. In such a population, even if a female can obtain a positive payoff from mating immediately, she should delay mating because her additional gain by reducing the chance of mating with a "bad" male exceeds her cost of delay.

The simplest interpretation of the parameters of the model is that they have fixed values. However, the analysis is compatible with a more liberal interpretation in which they can vary with variables that are objectively known by the male and female (i.e. are common knowledge), providing that the male and female have a degree of behavioural flexibility that allows them to condition their behaviour on these variables. These variables could include time of season, environmental variables such 
as temperature, and any objective and directly observable characteristics of the male or female that influence payoffs or the probability $P_{f}$ that a male will be good.

In this model "mating" represents an end-point of courtship in which fertilisation occurs with certainty or at least with a fixed probability. However, if the female can exercise significant post-copulatory choice, as may be the case in some arthropods (Carbone \& Rivera 1998; Edvardsson \& Arnqvist 2000), then copulation itself may be an important component of courtship effort. Duration of copulation may then act as a signal, with superior males (in the sense of preferable fertilisers, from a female's perspective) being willing to copulate for longer; it is then in the female's interest to select for fertilisation the sperm of males who will endure long copulations.

Although the female has the option of quitting without mating, and without incurring further costs, she makes use of this option only if she cannot obtain a positive payoff from participation. In an extended courtship equilibrium she does not make use of this option. As the model is currently formulated, removing the female's option of quitting would not affect the equilibrium behaviour of females or of males. The model would then represent a system in which a female's only possible response to a persistent male is the extent to which she delays mating, with delay involving energetic and other costs to her. This may approximate the situation in hermit crabs (Yamanoi et al. 2006) in which a female may delay mating with a pre-copulatory guarding male, and the male may - under competition from other males - quit guarding without mating. The biologically relevant question in relation to the present study is how far a male's ability to guard a female for a long time constitutes an indicator of his quality. If it does constitute such an indicator, then delaying mating with a guarding male is not 
simply a female's attempt to choose a different male. It may, at least in part, be a way of testing the quality of the male who is guarding her.

Various generalisations and extensions of this modelling approach are possible. Although the model captures the idea that the duration of a male's courtship effort is a useful signal to the female, in some biological species such as horned dung beetles (Kotiaho 2002) the intensity of the male's effort is also important. Representing this in a model would involve relaxation of the assumption of a fixed cost of courtship per unit time to the male; if applied to the present model this would greatly complicate the analysis.

Another possible generalization would relax the assumption of a homogeneous female population. The current model assumes that females are homogeneous in their costs and payoffs, resulting in them all having the same unique best response mating rate $\lambda$ to a given bad male quitting rate (Fig 3a). The bad male best response quitting rate against this is a step function (Fig 3b), and the existence of a point mass of homogeneous bad males then enables formation of an equilibrium with extended courtship in which the female mating rate is equal to the value $\omega_{\mathrm{B}}$ at which this step occurs. Relaxing the homogeneity assumption for the female population would result in females exhibiting instead a distribution of mating rates rather than a single mating rate. Male best response quitting rates to such a distribution then would vary continuously with male type, allowing extended courtship equilibria for a population in which male type varies continuously throughout its range. This development would require a significant extension of the analysis presented here. 
The model is a highly idealized representation of a single courtship encounter. It is not intended to represent the detailed courtship behaviour of any particular species. Rather, it is a generic model designed to shed light on the costs and benefits of extended-time courtship behaviour. For humans in particular, cognitive capacity and behavioural flexibility is vastly greater than is assumed in this simple model. People can use a range of subtle and variable signals, and cumulatively assess complex features of potential mating partners over time (Miller, 2000; Buss, 2003; Saad and Gill, 2003; Brase, 2006; Griskevicius et al, 2007). In addition, human courtship takes place (and probably always has taken place) within a complex social environment. Potentially this allows individual participation costs in courtship to be diminished, with honesty of signals sustained by social enforcement mechanisms (Lachman et al, 2001). Nevertheless, we believe that the simple model presented here captures a possible role for costs accumulated over time which may be important in extended courtship in several species, including humans. It may therefore help to explain long courtship in humans, and the folk wisdom that a woman is best advised not to sleep with a man on a first date.

\section{Acknowledgments}

We thank Mike Ritchie, Ray Heaton, Constantino Macias Garcia and three anonymous referees for comments and suggestions on this work. RMS was supported by the Centre for Mathematics and Physics in the Life Sciences and Experimental Biology (CoMPLEX) at UCL. PDS was supported by the London School of Economics. 


\section{References}

Alcock, J., 1998. Animal Behaviour: an Evolutionary Approach, $6^{\text {th }}$ ed. Sinauer, Sunderland Massachusetts.

Andersson, M., 1994. Sexual Selection. Princeton University Press, Princeton.

Andersson, M., Iwasa, Y., 1996. Sexual selection. Trends Ecol. Evol. 11, 53-58.

Brown, W.D., Kuns, M.M., 2000. Female Choice and the Consistency of Courtship Feeding in Black-horned Tree Crickets Oecanthus nigricornis Walker (Orthoptera: Gryllidae: Oecanthinae). Ethology 106, 543-557.

Buss, D.M, 2003. The evolution of desire: Strategies of human mating, $2^{\text {nd }}$ ed. New York: Basic Books.

Camerer, C., 1988. Gifts as economic signals and social symbols. American Journal of Sociology 94, S180-S214.

Carbone, S.S., Rivera, A.C., 1998. Sperm competition, cryptic female choice, and prolonged mating in the eucalyptus snout-beetle, Gonipterus Scutellatus (Coleoptera, Curculionidae). Etologia 6, 33-40.

Dall, S.R.X., Giraldeau, L.A., Olsson, O., McNamara, J.M., Stephens, D.W., 2005. Information and its use by animals in evolutionary ecology. Trends in Ecololgy and Evolution 20, 187-192. 
Dawkins, M.S., 1993. Are there general principles of signal design? Phil. Trans. R. Soc. Lond. B 340, 251-255.

Edvardsson, M., Arnqvist, G., 2000. Copulatory courtship and cryptic female choice in red flour beetles Tribolium castaneum. Proc. R. Soc. Lond. B 267, 559-563.

Frith, C.B., Beehler, B.M., 1998. The birds of paradise: Paradisaeidae. Oxford University Press, Oxford.

Grafen, A., 1990a. Sexual selection unhandicapped by the Fisher process. J. Theor. Biol. 144, 473-516.

Grafen, A., 1990b Biological signals as handicaps. J. Theor. Biol. 144, 517-546.

Griskevicius, V., Tybur, J.M., Sundie, J.M., Cialdini, R.B., Miller, G.F., Kendrick, D.T., 2007. Blatant Benevolence and Conspicuous Consumption: When Romantic Motives Elicit Strategic Costly Signals. Journal of Personality and Social Psychology, 93, 85-102.

Gwynne, D.T., 1984. Courtship feeding increases female reproductive success in bushcrickets. Nature 307, 361-363.

Johnstone, R.A., 1997. The evolution of animal signals. In: Behavioural ecology: an evolutionary approach, 4th ed. Eds: J.R. Krebs and N.B. Davies, pp 155-178. Oxford: Blackwell Scientific. 
Kondoh, M., 2001. Co-evolution of nuptial gift and female multiple mating resulting in diverse breeding systems. Evolutionary Ecology Research 3, 75-89.

Kotiaho, J.S., 2002. Sexual selection and condition dependence of courtship display in three species of horned dung beetles. Behavioural Ecology 13, 791-799.

Krakauer, D.C., Johnston, R.A., 1995. The Evolution of Exploitation and Honesty in Animal Communication: A Model Using Artificial Neural Networks. Phil. Trans. R. Soc. Lond. B 348, 355-361.

Krebs, J.R., Dawkins, R., 1984. Animal signals: mind-reading and manipulation. In: Behavioural ecology: an evolutionary approach, $2^{\text {nd }}$ ed. Eds: J.R. Krebs and N.B. Davies, pp 380-402. Oxford: Blackwell Scientific.

Lachman, M., Szamado, S., Bergstrom, C.T., 2001. Cost and conflict in animal signals and human language. Proc Natl Acad Sci USA 98, 13189-13194.

LeBas, N.R., Hockam, L.R., 2005. An invasion of cheats: the evolution of worthless nuptial gifts. Current Biology 15, 64-67.

Luttbeg, B., 1996. A comparative Bayes tactic for mate assessment and choice. Behavioural Ecology 7, 451-460. 
Maynard Smith, J., 1974. The theory of games and the evolution of animal conflicts. J. Theor. Biol. 47, 209-221.

Miller, G., 2000. The Mating Mind. Heinemann, London.

Moore, P.J., Moore, A.J., 2001. Reproductive aging and mating: The ticking of the biological clock in female cockroaches. PNAS 98, 9171-9176

Nisbet, J.C.T., 1973. Courtship-feeding, Egg-size and Breeding Success in Common Terns. Nature 241, 141-142.

Odendaal, F.J., Iwasa, Y., Ehrlich, P.R., 1985. Duration of Female Availability and Its Effect on Butterfly Mating Systems. Am. Nat. 125, 673-678.

Parker, G. A., 1982. Phenotype limited evolutionarily stable strategies. In Current Problems in Sociobiology (ed. B.R. Bertram, T. H. Clutton-Brock, R.I.M. Dunbar, D.I. Rubinstein, and R. Wrangham), pp 173-201. Cambridge: Cambridge University Press.

Pizzari, T., 2003. Food, vigilance, and sperm: The role of male direct benefits in the evolution of female preference in a polygamous bird. Behav. Ecol. 14, 593-601.

Pomiankowski, A., 1987. The costs of choice in sexual selection. J Theor Biol 128, 195-218. 
Roberts, G., 1998. Competitive altruism: From reciprocity to the handicap principle. Proc. R. Soc. Lond. B 265, 427-431.

Saad, G. and Gill, T., 2003. An evolutionary psychology perspective on gift-giving among young adults. Psychology \& Marketing, 20, 765-784.

Sozou, P.D., Seymour, R.M., 2005. Costly but worthless gifts facilitate courtship. Proc. R. Soc. Lond. B. 272, 1877-1884.

Spence, M., 1973. Job market signalling. Quarterly Journal of Economics 87, 355374.

Stalhandske, P., 2001. Nuptial gift in the spider Pisaura mirabilis maintained by sexual selection. Behav. Ecol. 12, 691-697.

Stalhandske, P., 2002. Nuptial gifts of male spiders function as sensory traps. Proc. R. Soc. Lond. B. 269, 905-908.

Tryjanowski, P., Hromada, M., 2005. Do males of the great grey shrike, Lanius excubitor, trade food for extrapair copulations? Animal Behaviour 69, 529-533.

Vahed, K., 1998. The function of nuptial feeding in insects: a review of empirical studies. Biol. Rev. 73, 43-78. 
Wedell, N., 1993. Spermatophore size in bushcrickets: comparative evidence for nuptial gifts as a sperm competition device. Evolution. 47, 1203-1212.

Yamanoi, T., Yoshino, K., Kon, K., Goshima, S., 2006. Delayed copulation as a means of female choice by the hermit crab Pagurus filholi. J Ethol 24, 213-218.

Zahavi, A., Zahavi, A., 1997. The handicap principle: A missing piece of Darwin's puzzle. Oxford University Press. 


\section{Appendix A: Evolutionary stability}

Consider an equilibrium $\left(q^{*}, \omega_{B}\right)$ (section 3.8). The female's best reply curve, defined for $q_{B}>\sigma_{B} /\left(1-P_{f}\right)$, is $\lambda=\lambda^{*}\left(q_{B}\right)$, given by (17). The male's best reply curve is $q_{B}^{*}(\lambda)=\infty$ for $\lambda<\omega_{B}$, and $q_{B}^{*}(\lambda)=0$ for $\lambda>\omega_{B}$. Then we have $\lambda^{*}\left(q^{*}\right)=\omega_{B}$ (section 3.3 and Fig 3).

Consider an evolutionary dynamics of the form:

$\dot{\lambda}=\left(\lambda^{*}\left(q_{B}\right)-\lambda\right) B_{F}\left(q_{B}, \lambda\right)$,

$\dot{q}_{B}=\left(\omega_{B}-\lambda\right) B_{M}\left(q_{B}, \lambda\right)$,

where the functions $B_{F}$ and $B_{M}$ are strictly positive in a neighbourhood of equilibrium, but are otherwise arbitrary. These dynamics have the property that they move $q_{B}$ and $\lambda$ in the direction of best reply at rates determined both by the size of the perturbation away from equilibrium, and by the factors $B_{F}$ and $B_{M}$.

The Jacobian matrix of the dynamics (A.1a, b) at the equilibrium $\left(q^{*}, \omega_{B}\right)$ is:

$$
J=\left(\begin{array}{cc}
-B_{F}^{*} & \lambda^{* \prime}\left(q^{*}\right) B_{F}^{*} \\
-B_{M}^{*} & 0
\end{array}\right)
$$


Thus, $\operatorname{Tr} J=-B_{F}^{*}<0$ and Det $=\lambda^{* \prime}\left(q^{*}\right) B_{F}^{*} B_{M}^{*}$. When $q^{*}=q_{0}^{*}$, we have $\lambda^{* \prime}\left(q_{0}^{*}\right)<0$

(Fig 3a), and hence DetJ $<0$. This shows that $\left(q_{0}^{*}, \omega_{B}\right)$ is unstable under the dynamics (A.1a, b). On the other hand, when $q^{*}=q_{1}^{*}$, we have $\lambda^{* \prime}\left(q_{1}^{*}\right)>0$ (Fig 3a), and hence $\operatorname{Det} J>0$. It follows that $\left(q_{1}^{*}, \omega_{B}\right)$ is locally asymptotically stable under the dynamics (A.1a, b).

\section{Appendix B: Equilibrium outcomes}

In this Appendix we prove the claims made in section 3.7. In particular, we construct the function $f\left(P_{f}\right)$, defined for $0 \leq P_{f}<1$, such that $\left(q_{B}, \lambda\right)=\left(q_{1}^{*}, \omega_{B}\right)$ is an extended courtship equilibrium for all parameter pairs $\left(P_{f}, \omega_{B}\right)$ with $\omega_{B}>f\left(P_{f}\right)$.

The bad male equilibrium quitting behaviour $q_{1}^{*}$ is the larger root of the equation $\lambda^{*}\left(q_{B}\right)=\omega_{B}$. From (17), this equilibrium condition yields the quadratic equation, $Q\left(q_{B}\right)=0$, where

$Q\left(q_{B}\right)=P_{f} \sigma_{B} q_{B}^{2}+\omega_{B}\left\{2 P_{f} \sigma_{B}-\omega_{B}\left(1-P_{f}\right)\right\} q_{B}+\omega_{B}^{2} \sigma_{B}$

The larger root of this quadratic, after some algebraic manipulation, can be written as

$$
q_{1}^{*}=\frac{\omega_{B}}{2 P_{f} \sigma_{B}}\left\{\left\{\omega_{B}-\left(2 \sigma_{B}+\omega_{B}\right) P_{f}\right\}+\sqrt{\left\{\omega_{B}^{2}-\left(2 \sigma_{B}+\omega_{B}\right)^{2} P_{f}\right\}\left(1-P_{f}\right)}\right\}
$$


It follows from (19) that the condition $q_{1}^{*}>\hat{q}_{B}$ for the female to obtain a positive payoff from this equilibrium behaviour, holds if and only if $\omega_{B}>\sigma_{G}$ and

$$
\begin{aligned}
\left\{\omega_{B}-\left(2 \sigma_{B}+\omega_{B}\right) P_{f}\right\} & +\sqrt{\left\{\omega_{B}^{2}-\left(2 \sigma_{B}+\omega_{B}\right)^{2} P_{f}\right\}\left(1-P_{f}\right)} \\
& >2\left(1-P_{f}\right) \frac{\sigma_{G}\left(\omega_{B}+\sigma_{B}\right)}{\left(\omega_{B}-\sigma_{G}\right)}-2 P_{f} \sigma_{B} .
\end{aligned}
$$

After some rearrangement, this inequality can be written as:

$$
\sqrt{\frac{\alpha^{2}-P_{f}}{1-P_{f}}}>\frac{2 \sigma_{G} \sigma_{B}+3 \sigma_{G} \omega_{B}-\omega_{B}^{2}}{\left(2 \sigma_{B}+\omega_{B}\right)\left(\omega_{B}-\sigma_{G}\right)},
$$

where $\alpha=\omega_{B} /\left(2 \sigma_{B}+\omega_{B}\right)$. Note that, from (18), $q_{1}^{*}$ exists only if $P_{f} \leq \alpha^{2}$, so the left-hand-side of (B.2) is well defined.

Clearly, the right hand side of (B.2) becomes negative if $\omega_{B}$ is sufficiently large, and in this case (B.2) is automatically satisfied. Indeed, as $\omega_{B} \rightarrow \infty, \alpha \rightarrow 1$ and so the left hand side $\rightarrow 1$, whereas the right hand side $\rightarrow-1$. The right hand side of (B.2) is negative for $\omega_{B}>\omega_{B}^{0}$, where $\omega_{B}^{0}=\frac{3}{2} \sigma_{G}\left\{1+\sqrt{1+8 \sigma_{B} / 9 \sigma_{G}}\right\}$, and for $\omega_{B}$ in this range $q_{1}^{*}$ is an ESS strategy for any $P_{f}$ in the interval $0 \leq P_{f} \leq \alpha^{2}$ for which it is defined. Thus, the boundary curve to this region, $P_{f}=\alpha^{2}$, is the curve $\omega_{B}=f\left(P_{f}\right)$, where

$$
f\left(P_{f}\right)=2 \sigma_{B} \frac{\sqrt{P_{f}}}{1-\sqrt{P_{f}}} .
$$


This is a monotonically increasing function of $P_{f}$ defined for $P_{f}>P_{f}^{0}$, where $P_{f}^{0}=\alpha^{2}\left(\omega_{B}^{0}\right)$. Clearly $f\left(P_{f}\right) \rightarrow \infty$ as $P_{f} \rightarrow 1$.

For any $\omega_{B}$, the maximum value of the left hand side of (B.2) is attained when $P_{f}=0$. Thus, (B.2) cannot hold for values of $\omega_{B}$ for which the right hand side is larger than $\alpha$. This implies that (B.2) can hold only for values of $\omega_{B}$ satisfying $\omega_{B}^{2}-2 \sigma_{G} \omega_{B}-\sigma_{G} \sigma_{B}>0$. That is, for values satisfying $\omega_{B}>\hat{\omega}_{B}$, where $\hat{\omega}_{B}=\sigma_{G}+\sqrt{\sigma_{G}\left(\sigma_{G}+\sigma_{B}\right)}$, as given in (21). Furthermore, equality holds in (B.2) (i.e. $\left.q_{1}^{*}=\hat{q}_{B}\right)$ when $\left(P_{f}, \omega_{B}\right)=\left(0, \hat{\omega}_{B}\right)$.

Note that $\omega_{B}^{0}>\hat{\omega}_{B}$, and so it remains to determine conditions under which (B.2) holds for $\omega_{B}$ in the range $\hat{\omega}_{B}<\omega_{B} \leq \omega_{B}^{0}$. Let $\beta$ denote the right hand side of (B.2). Then $0 \leq \beta<\alpha$ for $\omega_{B}$ in this range, and $q_{1}^{*} \geq \hat{q}_{B}$ holds if and only if $\sqrt{\alpha^{2}-P_{f}} \geq \beta \sqrt{1-P_{f}}$; i.e. $P_{f} \leq g\left(\omega_{B}\right)$ where $g\left(\omega_{B}\right)=\frac{\alpha^{2}-\beta^{2}}{1-\beta^{2}}=1-\frac{1-\alpha^{2}}{1-\beta^{2}}$.

As shown above, we have $g\left(\hat{\omega}_{B}\right)=0$ and $g\left(\omega_{B}^{0}\right)=P_{f}^{0}$. A calculation from the definitions of $\alpha$ and $\beta$ yields the explicit formula: 


$$
g\left(\omega_{B}\right)=1-\left(\frac{\sigma_{B}}{\sigma_{G}+\sigma_{B}}\right) \frac{\left(\omega_{B}-\sigma_{G}\right)^{2}}{\omega_{B}\left(\omega_{B}-2 \sigma_{G}\right)} .
$$

[Observe that $\hat{\omega}_{B}>2 \sigma_{G}$ so that the denominator on the right is positive for $\left.\omega_{B} \geq \hat{\omega}_{B}.\right]$ Using (B.4), the function $P_{f}=g\left(\omega_{B}\right)$ can be inverted to obtain $\omega_{B}=f\left(P_{f}\right)$, defined for $0 \leq P_{f} \leq P_{f}^{0}$, and given explicitly by:

$$
f\left(P_{f}\right)=\sigma_{G}\left\{1+\sqrt{\frac{1-P_{f}}{P_{f}^{*}-P_{f}}}\right\},
$$

with $P_{f}^{*}=\sigma_{G} /\left(\sigma_{G}+\sigma_{B}\right)$, as in (20). This function is monotonically increasing in $P_{f}$, and satisfies $f(0)=\hat{\omega}_{B}$ and $f\left(P_{f}^{0}\right)=\omega_{B}^{0}$.

We have now defined a continuous, monotonically increasing function, $\omega_{B}=f\left(P_{f}\right)$, given by (B.3) for $P_{f}>P_{f}^{0}$, and by (B.5) for $0 \leq P_{f} \leq P_{f}^{0}$. Thus, $f\left(P_{f}\right)$ is defined for all $P_{f}$ in the range $0 \leq P_{f}<1$. It also satisfies $f(0)=\hat{\omega}_{B}$ and $f\left(P_{f}\right) \rightarrow \infty$ as $P_{f} \rightarrow 1$. Furthermore, $\left(q_{B}, \lambda\right)=\left(q_{1}^{*}, \omega_{B}\right)$ is an ESS for $\omega_{B}>f\left(P_{f}\right)$. This establishes the claims of section 3.7.

\section{Appendix C: Extended male type spectrum}

Throughout the main text of this paper, for ease of exposition we have assumed that males have one of two possible types, "good" $(G)$ or "bad" $(B)$. In this appendix we consider the effect of an arbitrary number of discrete male types. 
Assume that a male's type is described by a variable $g$ taking discrete values. This variable represents male "goodness": a higher value of $g$ means that the male is in better condition or has a stronger intention/capacity to provide paternal care (cf. section 2.2). We assume that a male's payoff from mating is always positive and is a monotonically increasing function of $g$. A female's expected payoff from mating is also monotonically increasing in $g$, but may be positive or negative depending on $g$.

Suppose that at equilibrium the males who participate in courtship are of types 0,1 , $\ldots, n, \ldots$, in ascending order of goodness; i.e. $g_{n+1}>g_{n}$. This leaves open the possibility that there are also male types in the population who do not attempt courtship but instead always quit immediately - for example, if they are not (yet) in sufficiently good condition, or have not acquired the necessary resources to carry the cost of courtship. In an extended courtship equilibrium the female's payoff from mating must be negative if the courting male is of type 0 , and must be positive if the male is sufficiently good. If the female received a negative payoff from mating with all types of courting male, her best strategy against any male would be to quit immediately, and if she received a positive payoff from mating with all types of courting male, her best strategy would be to mate immediately; either way there would be no extended courtship.

A female's strategy is given by a quitting rate $\theta$ and a mating rate $\lambda$. It is straightforward to show, using the argument of section 3.3, that if the female engages in courtship at all (i.e. if she does not quit immediately), then she should never quit. 
That is, $\theta=0$. Thus, if extended courtship occurs, a female's strategy is specified only by a mating rate $\lambda$.

Let $\omega_{n}$ be the ratio, for a male of type $n$, of his cost per unit time of participation in courtship to his gain from mating (cf. (8) and Table 1). Since male payoffs from mating are positive and monotonically increasing and cost $x$ per unit time is constant ${ }^{2}$, it follows that $\omega_{n}$ is positive and monotonically decreasing with $n$, i.e. better male types have lower $\omega$-values. Suppose the female has a positive and finite mating rate $\lambda$. From the analysis of section 3.3, the best response of a male of type $n$ is to quit immediately if $\omega_{n}>\lambda$, and never to quit if $\omega_{n}<\lambda$ (cf. Table 2). If $\omega_{n}$ is exactly equal to $\lambda$ then all quitting rates are equal best responses to $\lambda$.

For a male of type 0 to participate in courtship implies $\omega_{0} \leq \lambda$. It follows that all other male types $n=1,2,3 \ldots$ participating in courtship must satisfy $\omega_{n}<\lambda$, so types which are better than type 0 will court indefinitely without quitting. If it were also the case that $\omega_{0}<\lambda$, then a type 0 male would also never quit, giving a situation in which no courting male quits. The duration of courtship would then no longer act as a signal of a male's type; a female's best response would be to either mate immediately or quit immediately, and so there would be no extended courtship. If follows that an equilibrium with extended courtship must satisfy $\omega_{0}=\lambda$, so that for males of type 0 all quitting rates are an equal best response to $\lambda$. This leads to an outcome in which males of type 0 have a finite quitting rate (cf. case 4 in table 2), and all other males

\footnotetext{
${ }^{2}$ As for the binary case it can alternatively be assumed that all males have the same payoff from mating but better males have a lower cost per unit time for courtship (see footnote 1). The results depend on the ratio $\omega$ of the cost per unit time of courtship to the benefit from mating decreasing as goodness $g$ increases.
} 
who participate in courtship will court indefinitely. Note, however, that the population could additionally contain males who are worse (i.e. lower $g$ ) than type $0^{3}$ : such males will not participate in courtship, but instead will quit immediately on encountering a female.

The form of the equilibrium is essentially the same as for the binary case. Males of type 0 correspond to "bad" males: they give the female a negative payoff from mating and have a finite quitting rate. Other male types that participate in courtship can be regarded collectively as "good" males: these court indefinitely. This does not imply that a male type who does not quit necessarily gives the female a positive payoff from mating, but rather that the female can only condition (by delaying mating) on whether or not the male has a positive quitting rate, and that her expected payoff from mating with a male who does not quit is positive.

We can formally redefine the relevant parameters of the binary analysis for the case of multiple male types participating in courtship. Let the proportion of males who participate in courtship and are of type $n$ be $p_{n}$. Let $F_{n}$ be a female's payoff from mating with a male of type $n$, and $M_{n}$ be the payoff to a male of type $n$ from mating. Then the relevant parameters become:

$$
\begin{aligned}
& P_{f}=\sum_{n \geq 1} p_{n}, \text { with } 1-P_{f}=p_{0}, \\
& A_{f}=\frac{\sum_{n \geq 1} p_{n} F_{n}}{P_{f}}
\end{aligned}
$$

\footnotetext{
${ }^{3}$ We assume that a male with lower goodness than males of type 0 would obtain a payoff from mating which is positive but lower than the payoff for a male of type 0 , and hence would have an $\omega$-value greater than $\omega_{0}$.
} 


$$
\begin{aligned}
& C_{f}=-F_{0}, \\
& D_{m}=M_{0} .
\end{aligned}
$$

The analysis now proceeds exactly as for the binary case. Note that for the binary case there is a single payoff from mating for a good male, $A_{m}$, whereas for multiple male types this varies with type. However, in the binary case $A_{m}$ drops out of the analysis as long as $A_{m}>D_{m}$. In the case of multiple male types we have $M_{n}>M_{0}$ for all $n>0$, resulting in the same behaviour, i.e. good males do not quit.

In conclusion, the model with several discrete male types yields an outcome that in all essential respects is the same as that of the binary model. In particular, at an equilibrium with extended courtship, a female never quits, a male of type $n>0$ never quits, and Nash equilibria are determined by the quitting strategy of bad males, $q_{B}=q_{0}$. 


\section{FIGURE LEGENDS}

Fig 1. A possible game tree showing the moves in a 'round' of length $\delta t$. Each round is preceded by an assessment by the female of the male's type. In fact, to first order in $\delta t$, the order in which the moves are made is irrelevant.

Fig 2. The female's best reply mating frequency function $\lambda^{*}\left(q_{B}\right)$ to a bad male's quitting strategy $q_{B}$, given by (17), showing the possible Nash equilibria $q_{B}=q_{0}^{*}$ and $q_{B}=q_{1}^{*}$. This function is defined for $q_{B}>\sigma_{B} /\left(1-P_{f}\right)$. The grey-shaded region to the left in each panel is the region $0 \leq q_{B} \leq \sigma_{B} /\left(1-P_{f}\right)$, in which the female's best reply is either to quit immediately (if $E_{\text {prior }}^{F}<0$ ) or to mate immediately (if $E_{\text {prior }}^{F}>0$ ). For $q_{i}^{*}$ to define a Nash equilibrium requires that $q_{i}^{*}>\hat{q}_{B}$, the threshold given by (19). A heavy black point indicates that this condition is satisfied; a grey filled circle indicates that it is not satisfied. The cases shown are: $(\boldsymbol{a}) q_{0}^{*}$ and $q_{1}^{*}$ are both Nash equilibria. Here $\hat{q}_{B}<\sigma_{B} /\left(1-P_{f}\right)$. Parameters are: $P_{f}=0.375, \sigma_{G}=1, \sigma_{B}=2, \omega_{B}=8$. (b) $q_{0}^{*}$ and $q_{1}^{*}$ are both Nash equilibria, but with $\hat{q}_{B}>\sigma_{B} /\left(1-P_{f}\right)$. Parameters are: $P_{f}=0.286, \sigma_{G}=1, \sigma_{B}=2, \omega_{B}=4.7$. (c) $q_{1}^{*}$ is a Nash equilibrium, but $q_{0}^{*}$ is not. Parameters are: $P_{f}=0.667, \sigma_{G}=5, \sigma_{B}=1, \omega_{B}=13 .(d)$ Neither $q_{0}^{*}$ nor $q_{1}^{*}$ is a Nash equilibrium. Parameters are: $P_{f}=0.667, \sigma_{G}=5, \sigma_{B}=1, \omega_{B}=11$.

Fig 3. Best reply curves: a) for the female's mating frequency best response $\lambda=\lambda^{*}\left(q_{B}\right)$ to the male's quitting strategy $\left.q_{B} ; \mathbf{b}\right)$ for the male's quitting frequency 
best response $q_{B}=q^{*}(\lambda)$ to the female's mating strategy $\lambda$. The two possible Nash equilibria $\left(q_{B}, \lambda\right)=\left(q_{0}^{*}, \omega_{B}\right)$ and $\left(q_{B}, \lambda\right)=\left(q_{1}^{*}, \omega_{B}\right)$ are indicated in (a).

Fig 4. Arrows indicate the direction of motion around the equilibrium $\left(q_{B}, \lambda\right)=\left(q_{1}^{*}, \omega_{B}\right)$ when evolutionary pressure moves points $\left(q_{B}, \lambda\right)$ in the direction of best replies. The heavy curve is the female best reply function $\lambda=\lambda^{*}\left(q_{B}\right)$.

Fig 5. Regions of the $\left(P_{f}, \omega_{B}\right)$-plane showing various possible equilibrium outcomes. Region A (dark grey): The region in which $\left(q_{B}, \lambda\right)=\left(q_{1}^{*}, \omega_{B}\right)$, the evolutionarily stable equilibria with extended courtship, exist. This is bounded by the solid curve $\omega_{B}=f\left(P_{f}\right)$, as described in the text. Region B (light grey): The region in which the female quits immediately and no mating occurs. The dashed curve is determined by the threshold (18), $P_{f}=\omega_{B}^{2} /\left(2 \sigma_{B}+\omega_{B}\right)^{2}$. In the region to the left of this curve $q_{1}^{*}$ exists, but the strategy $\operatorname{pair}\left(q_{B}, \lambda\right)=\left(q_{1}^{*}, \omega_{B}\right)$ does not yield a positive payoff to the female $\left(\hat{q}_{B}>q_{1}^{*}\right)$. To the right of the dashed curve there are no solutions of $\lambda^{*}\left(q_{B}\right)=\omega_{B}$. Region $\mathbf{C}$ (white): In the region $P_{f}>P_{f}^{*}$ the female can obtain a positive payoff from mating immediately. This is the unique equilibrium outcome where it does not overlap with region A. Region D (very dark grey): The female payoff is positive from both mating immediately and from extended courtship. Her payoff from the latter is greater than from the former. Fixed parameters used are: $\sigma_{G}=5, \sigma_{B}=1$. 


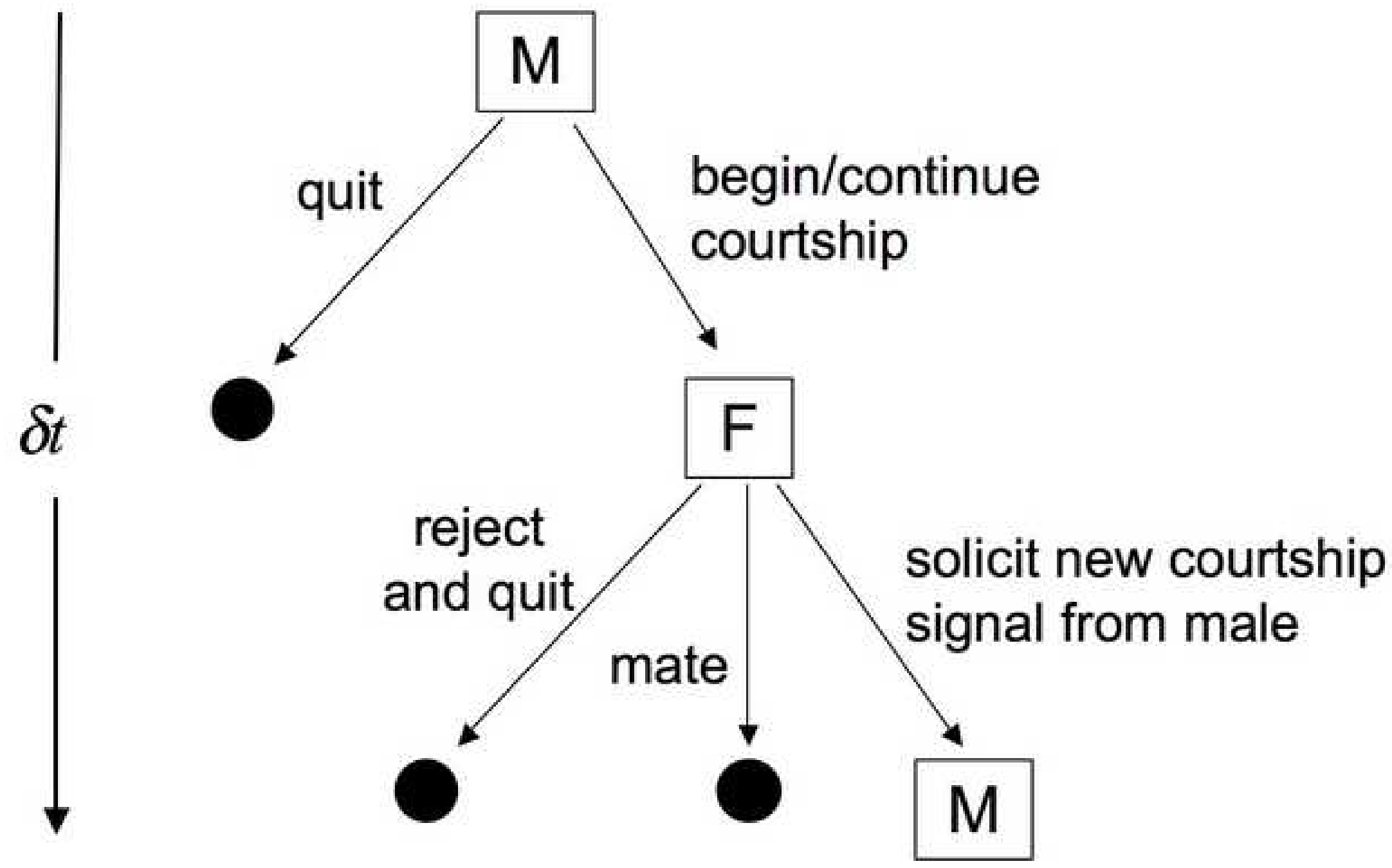

KEY: $=$ Game ends 

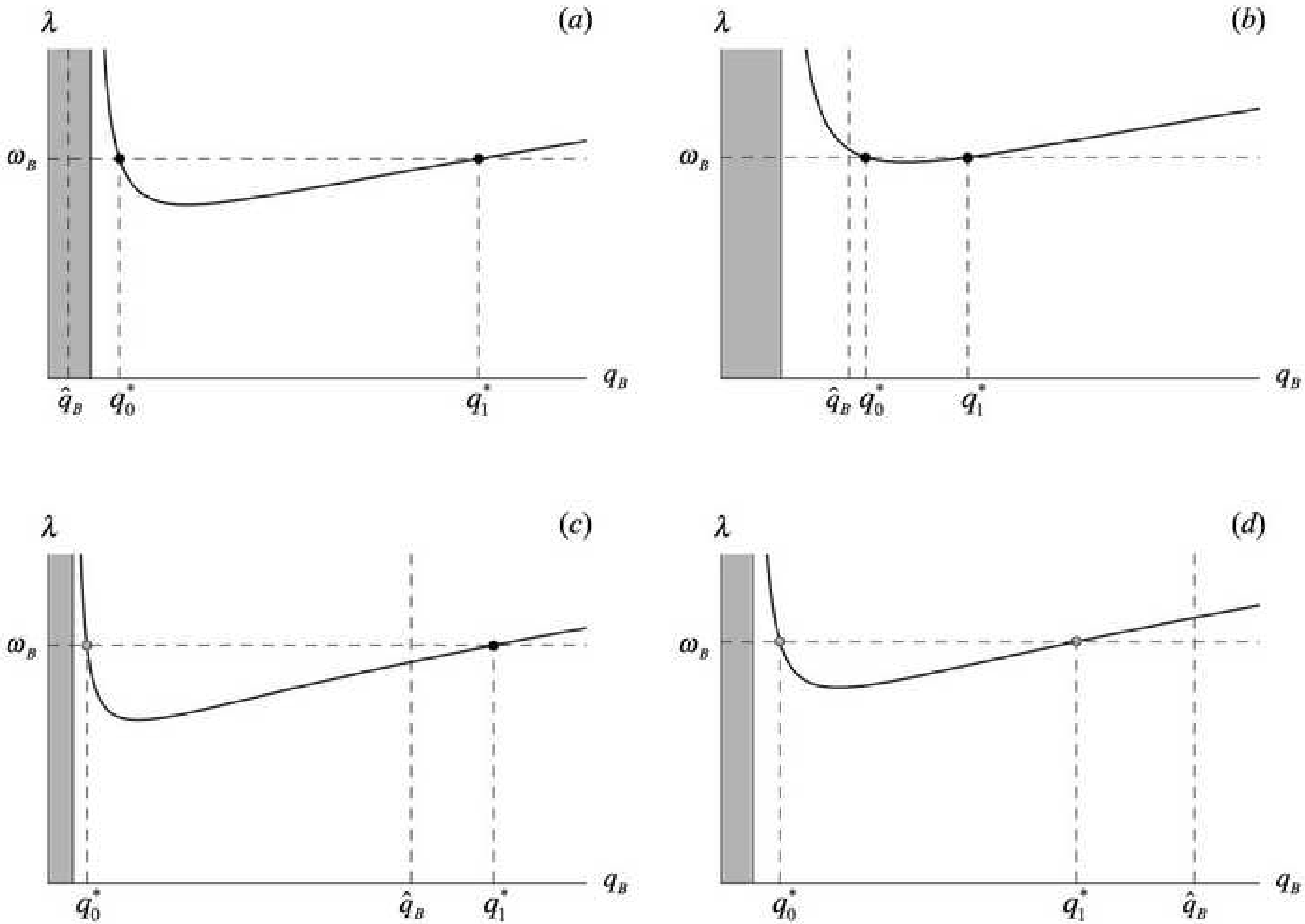
Click here to download high resolution image
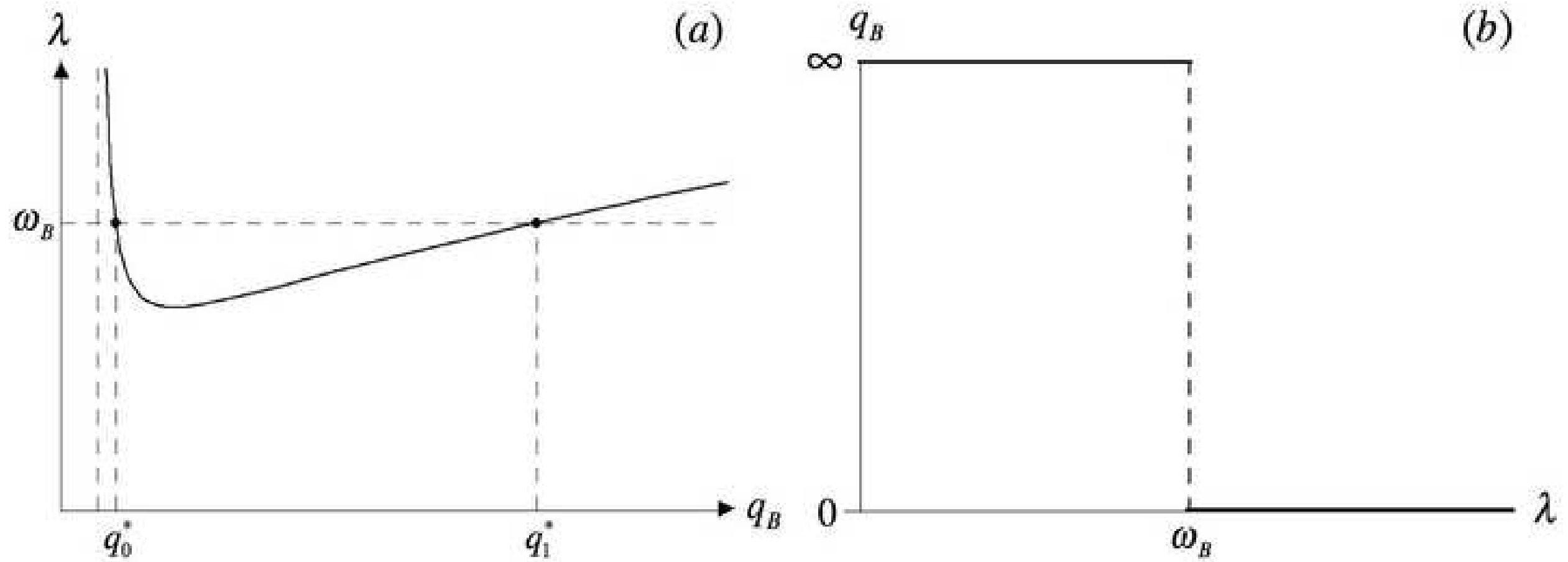


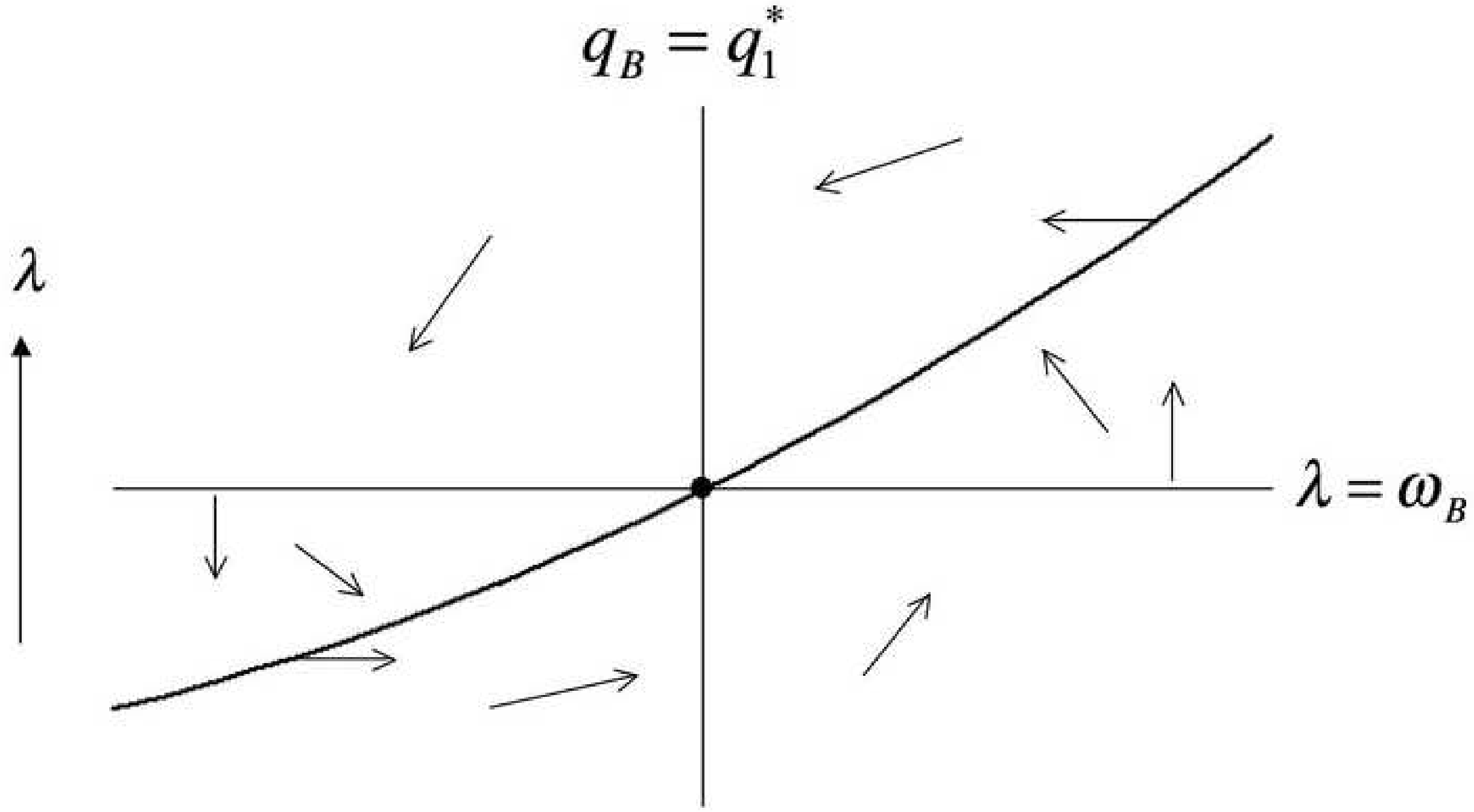




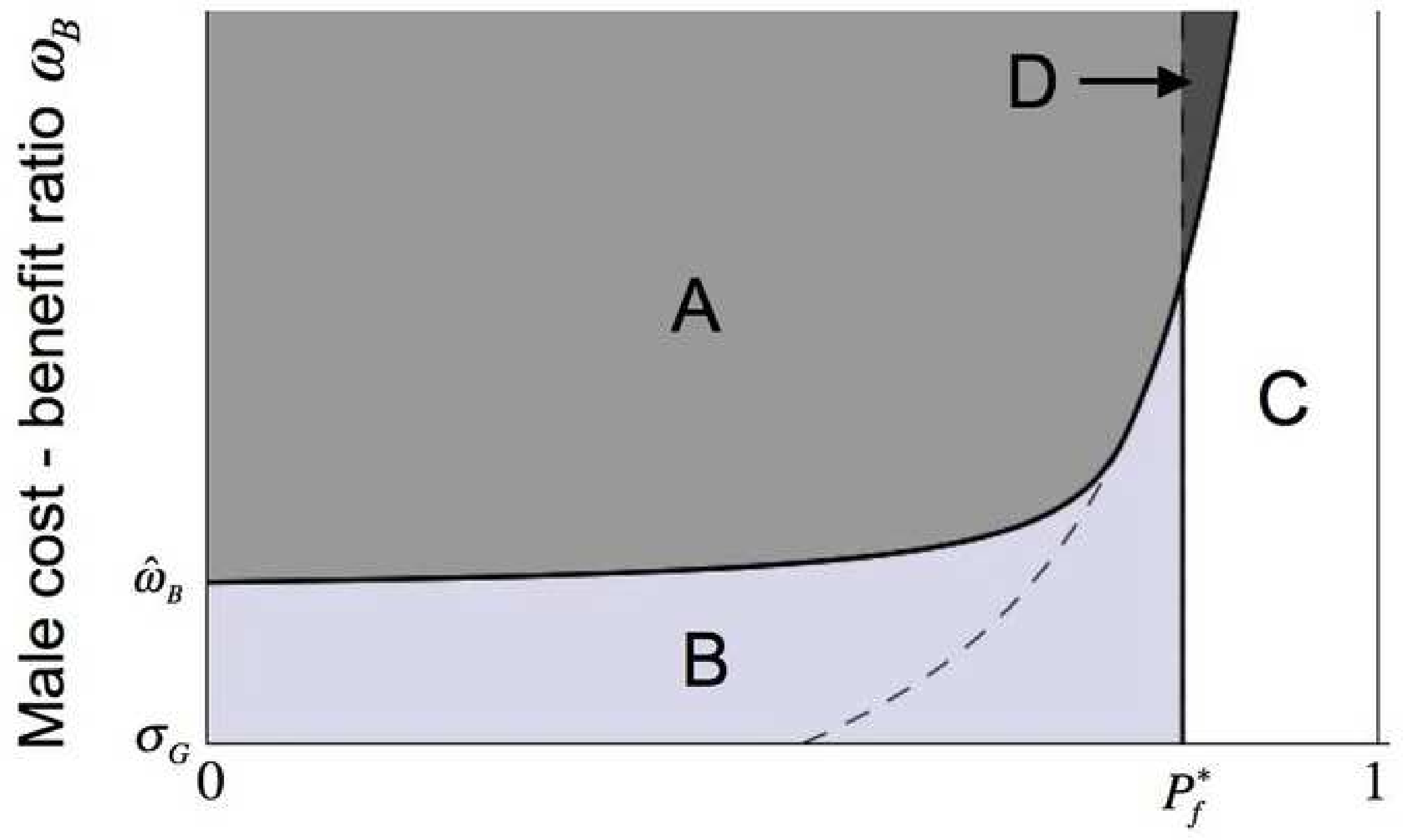

Probability that male is Good $P_{f}$ 